DOI 10.4171/JEMS/258

Giuseppe Mingione

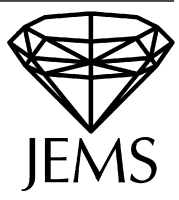

\title{
Gradient potential estimates
}

Received December 5, 2008 and in revised form June 15, 2009

Abstract. Pointwise gradient bounds via Riesz potentials like those available for the Poisson equation actually hold for general quasilinear equations.

\section{Introduction}

The aim of this paper is twofold; first, we prove a somehow surprising result, asserting the possibility of giving pointwise gradient estimates for solutions of non-linear, nonhomogeneous elliptic equations, via usual linear Riesz potentials, exactly as it happens for the Poisson equation via representation formulas. This fact is in turn achieved via a "fractional variation" of De Giorgi's iteration technique, whose presentation is the other aim of the paper. This method could be useful in other contexts, as we shall explain below, since it proposes to iterate level sets of solutions via fractional derivatives also when dealing with integer order equations, which are, on the other hand, "non-differentiable" in the classical sense of so called strong solutions. Finally, we demonstrate applications to the proof of optimal Lipschitz continuity criteria, and to the inference of optimal local estimates.

\subsection{Linear representation}

We shall first consider the simpler case of quasilinear equations of the type

$$
-\operatorname{div} a(D u)=\mu \quad \text { in } \Omega,
$$

where $\mu$ is in the most general case a signed Radon measure with finite total mass:

$$
|\mu|(\Omega)<\infty
$$

defined on the open bounded and Lipschitz domain $\Omega \subset \mathbb{R}^{n}, n \geq 2$; we of course denote by $|\mu|$ the total variation of $\mu$ and for the rest of the paper we shall consider the measure $\mu$ as defined on the whole space $\mathbb{R}^{n}$ by just letting $\mu \equiv 0$ outside $\Omega$. Here by solution we initially mean a function $u \in W^{1,1}(\Omega)$ such that 1.1 holds in the usual distributional

G. Mingione: Dipartimento di Matematica, Università di Parma, Viale G. P. Usberti 53/a, Campus, 43100 Parma, Italy; e-mail: giuseppe.mingione@ unipr.it 
sense. We shall always consider the following growth and ellipticity assumptions on the $C^{1}$-vector field $a: \mathbb{R}^{n} \rightarrow \mathbb{R}^{n}$ :

$$
\nu|\lambda|^{2} \leq\langle\partial a(z) \lambda, \lambda\rangle, \quad|\partial a(z)| \leq L, \quad|a(0)| \leq L,
$$

which are satisfied whenever $z, \lambda \in \mathbb{R}^{n}$, with some $0<v \leq 1 \leq L$. The linear case $a(z)=z$ leads to the Poisson equation

$$
-\Delta u=\mu \quad \text { in } \mathbb{R}^{n},
$$

with $u \equiv 0$ at infinity, for which, due to the use of classical representation formulas, we can in particular bound the gradient via the use of Riesz potentials

$$
I_{\beta}(\mu)(x):=\int_{\mathbb{R}^{n}} \frac{d \mu(y)}{|x-y|^{n-\beta}}, \quad \beta \in(0, n),
$$

as follows:

$$
|D u(x)| \leq c I_{1}(|\mu|)(x) .
$$

Estimate (1.5) eventually leads to all type of gradient estimates in rearrangement invariant spaces, since by (1.5) the whole issue reduces to the study of the behavior of the Riesz potential $I_{1}$, which is in turn well-known [19]. Inequality (1.5) cannot be derived in this way for non-linear equations of the type (1.1), since no representation formulae are available. In this paper we show that pointwise estimates via linear potentials of the type (1.5) still hold in the quasilinear case (1.1). Since we are dealing with local solutions in bounded domains, we use a localized version of the Riesz potential [1.5] as in [4, 25], i.e.

$$
\mathbf{I}_{\beta}^{\mu}\left(x_{0}, R\right):=\int_{0}^{R} \frac{\mu\left(B\left(x_{0}, t\right)\right)}{t^{n-\beta}} \frac{d t}{t}, \quad \beta \in(0, n),
$$

with $B\left(x_{0}, t\right)$ being the open ball centered at $x_{0}$ with radius $t$. Notice that, in the case of non-negative measures $\mu$, by [4, Lemma 3.1.1] we have

$$
\mathbf{I}_{\beta}^{\mu}\left(x_{0}, R\right) \lesssim \int_{B_{R}\left(x_{0}\right)} \frac{d \mu(y)}{\left|x_{0}-y\right|^{n-\beta}}=I_{\beta}\left(\mu\left\llcorner B\left(x_{0}, R\right)\right)\left(x_{0}\right) \leq I_{\beta}(\mu)\left(x_{0}\right) .\right.
$$

The first result we present, which for the sake of simplicity we here state in the form of an a priori estimate for more regular solutions, now reads as follows:

Theorem 1.1 (Riesz potential bound). Let $u \in C_{\mathrm{loc}}^{1, \alpha}(\Omega), \alpha>0$, be a solution to the equation (1.1), under the assumptions 1.2 and with $\mu \in L^{\infty}(\Omega)$. Then there exists a constant $c \equiv c(n, L / v)$ such that whenever $\xi \in\{1, \ldots, n\}$ the pointwise estimate

$$
\left|D_{\xi} u\left(x_{0}\right)\right| \leq c f_{B\left(x_{0}, R\right)}\left|D_{\xi} u\right| d x+c \mathbf{I}_{1}^{|\mu|}\left(x_{0}, 2 R\right)
$$

holds whenever $B\left(x_{0}, 2 R\right) \subseteq \Omega$. 
The proof is in Section 3. An approximation procedure then allows us to remove the extra regularity assumption $\mu \in L^{\infty}$ _note that from this it follows $u \in C^{1, \alpha}$. At this stage the notion of solution considered must be specified according to the regularity of the right hand side $\mu$ : if $\mu \in W^{-1,2}$ we shall consider usual energy solutions, otherwise so called very weak solutions will come into play, i.e. solutions which not necessarily belong to the natural energy spaces $W^{1,2}$; see Section 4 and Theorem 4.1 below.

The second result, which is the main one of the paper, and which actually embraces Theorem 1.1, allows us to deal with more general equations of the type

$$
-\operatorname{div} a(x, D u)=\mu \quad \text { in } \Omega,
$$

where, for every choice of $x \in \Omega$, the vector field $z \mapsto a(x, z)$ satisfies $(1.2)$; we assume the Hölder continuity with respect to coefficients - complemented by a natural growth condition-that is, we assume that

$$
\left|a\left(x_{1}, z\right)-a\left(x_{2}, z\right)\right| \leq L_{1}\left|x_{1}-x_{2}\right|^{\sigma}(s+|z|), \quad|a(x, 0)| \leq L s
$$

for some $\sigma \in(0,1]$, whenever $x_{1}, x_{2} \in \Omega$ and $z \in \mathbb{R}^{n}$, with some $L_{1} \geq L$ and $s \geq 0$. The statement, again presented as an a priori estimate, and from which actually Theorem 1.1 follows in the case of no dependence on $x$, is

Theorem 1.2 (Hybrid bound). Let $u \in C_{\mathrm{loc}}^{1, \alpha}(\Omega), \alpha>0$, be a solution to the equation (1.9), under the assumptions (1.2) and (1.10), with $\mu \in L^{\infty}(\Omega)$. Then there exists a constant $c \equiv c\left(n, L / v, L_{1}, \sigma\right)$ such that whenever $\xi \in\{1, \ldots, n\}$ the pointwise estimate

$\left|D_{\xi} u\left(x_{0}\right)\right| \leq c f_{B\left(x_{0}, R\right)}\left|D_{\xi} u\right| d x+c \mathbf{I}_{1}^{|\mu|}\left(x_{0}, 2 R\right)+c L_{1}\left[\mathbf{I}_{\sigma}^{|D u|}\left(x_{0}, 2 R\right)+\frac{s}{\sigma} R^{\sigma}\right]$

holds whenever $B\left(x_{0}, 2 R\right) \subseteq \Omega$.

The proof is in Section 3. The effect of $x$-dependence is the additional pointwise quantity involving the gradient on the right hand side in (1.11). This is anyway not bad since the function $x \mapsto \mathbf{I}_{\sigma}^{|D u|}(x, 2 R)$ turns out to be obviously more regular than $|D u|$, this being essentially the smoothing effect of convolution with the kernel $|x|^{\sigma-n}$. For this reason estimate (1.11) is as good as 1.8 in order to prove bounds in various function spaces; see Section 4.3 below. Notice that the integrability of such a kernel is precisely dictated by the Hölder regularity of $x \mapsto a(x, \cdot)$.

\subsection{Fractional Caccioppoli inequalities}

The method of proof chosen here features the implementation of a fractional De Giorgi type iteration, which we think is of independent interest, devised to apply to problems with lack of full differentiability, like those involving measure data and/or non-differentiable coefficients. The starting point for proving gradient estimates for solutions to quasilinear elliptic equations is to differentiate the equation considered, after establishing that the second derivatives $D^{2} u$ exist, so that the components of $D u$ solve new, "linearized" 
equations. Then gradient bounds usually follow by applying an iteration procedure. We shall here focus on the one introduced by De Giorgi [13], where a control of the decay rate of the level sets of the gradient of solutions finally leads to the desired bounds. A crucial point is that the level set control is achieved by controlling in turn $D u$ by $D^{2} u$ via so called Caccioppoli inequalities. On the other hand this is not always possible for problems with bad right hand side and/or non-differentiable coefficients as in (1.9), as solutions are not twice differentiable in general. The idea at this point is to prove the existence of higher derivatives of fractional order, roughly denoted as $D^{1+\sigma_{0}} u$, and to control the level sets of $D u$ via $D^{1+\sigma_{0}} u$ instead of $D^{2} u$. The related iteration works thanks to a neat fractional Caccioppoli inequality on level sets of the gradient which can be considered as a main result of the paper, and which for simplicity we state for the case (1.1):

Theorem 1.3 (Fractional Caccioppoli inequality). Under the assumptions of Theorem 1.1. whenever $\xi \in\{1, \ldots, n\}, k \geq 0$, and for any ball $B_{R} \subseteq \Omega$, the inequality

$$
\left[\left(\left|D_{\xi} u\right|-k\right)_{+}\right]_{\sigma_{0}, 1 ; B_{R / 2}} \leq \frac{c}{R^{\sigma_{0}}} \int_{B_{R}}\left(\left|D_{\xi} u\right|-k\right)_{+} d x+\frac{c R|\mu|\left(B_{R}\right)}{R^{\sigma_{0}}}
$$

holds for every $\sigma_{0}<1 / 2$, where the constant c depends only on $n, L / v, \sigma_{0}$.

The proof is in Section 2.4 We have again stated the inequality for more regular solutions, something that can be avoided by means of a suitable approximation argument; needless to say, what matters here is the precise form of the a priori estimate (see Theorem 4.2 below). Here, following standard notation, for a general function $w$ we write $(w-k)_{+}:=\max \{w-k, 0\}$, while $\left[\left(\left|D_{\xi} u\right|-k\right)_{+}\right]_{\sigma_{0}, 1 ; B_{R / 2}}$ denotes the $L^{1}$-norm of the " $\sigma_{0}$-order derivative" of the truncated gradient $\left(\left|D_{\xi} u\right|-k\right)_{+}$; see 2.1 below for fractional derivatives. The key ingredient in De Giorgi's approach to regularity of solutions to linear elliptic equations of the type

$$
\operatorname{div}(A(x) D w)=0
$$

where $A(x)$ is a strictly elliptic matrix with bounded and measurable entries, is the following Caccioppoli inequality on level sets:

$$
\left[(w-k)_{+}\right]_{1,2 ; B_{R / 2}}^{2} \equiv \int_{B_{R / 2}}\left|D(w-k)_{+}\right|^{2} d x \leq \frac{c}{R^{2}} \int_{B_{R}}(w-k)_{+}^{2} d x .
$$

The essential feature of the last inequality is that it allows one to control the gradient $D w$ by the solution $w$ itself, on level sets. In our case-we are looking for the regularity of $w \equiv\left|D_{\xi} u\right|$-it is clear that inequality 1.12 is the natural fractional order version of (1.14). Ultimately, comparing (1.12) and (1.14) with $w \equiv\left|D_{\xi} u\right|$, Theorem 1.3 tells us that for quasilinear equations Caccioppoli's inequalities are a robust tool that holds at intermediate regularity levels: full derivatives can be replaced by fractional ones, the $L^{2}$-norm by the (weaker) norm of $L^{1}$. As is natural in these contexts, the Caccioppoli inequality 1.3 contains all the information leading to (1.8): 
Theorem 1.4 (De Giorgi's fractional iteration). Let $w \in L^{1}(\Omega)$ and $\mu$ be a Radon measure; let $\sigma_{0} \in(0,1)$ and $L \geq 1$ and assume that for every ball $B_{R} \subseteq \Omega$ and all $k \geq 0$,

$$
\left[(|w|-k)_{+}\right]_{\sigma_{0}, 1 ; B_{R / 2}} \leq \frac{L}{R^{\sigma_{0}}} \int_{B_{R}}(|w|-k)_{+} d x+\frac{L R|\mu|\left(B_{R}\right)}{R^{\sigma_{0}}} .
$$

Then

$$
\left|w\left(x_{0}\right)\right| \leq c f_{B\left(x_{0}, R\right)}|w| d x+c \mathbf{I}_{1}^{|\mu|}\left(x_{0}, 2 R\right)
$$

whenever $B\left(x_{0}, 2 R\right) \subset \Omega$ and $x_{0}$ is a Lebesgue point of $w$, for a constant $c$ which only depends on $n, L, \sigma_{0}$.

Theorem 1.1 can indeed be obtained as a combination of Theorems 1.3 and 1.4 , applied to $w:=\left|D_{\xi} u\right|$. Such a procedure is typical in the regularity theory starting from the fundamental paper of De Giorgi [13]: the property of being a solution is used to prove that the function in question satisfies a Caccioppoli type inequality as for instance (1.15); then further regularity follows using this sole property.

\subsection{The sharp Lipschitz criterion}

A main consequence of estimate 1.11 is the local Lipschitz continuity result of Theorem 1.5 below, which allows one to get for general non-linear elliptic equations a sharp criterion in terms of Riesz potentials which is classical for the Poisson equation. Indeed, as shown in [10], when considering $\left[1.3\right.$, the gradient $D u$ is bounded iff $\mathbf{I}_{1}^{|\mu|}(\cdot, R) \in L^{\infty}$; here the same sharp criterion is obtained for general non-linear equations. The notion of solution we are referring to is the one, typical of measure data, of Solution Obtained by Limit of Approximations (SOLA), and it coincides with usual weak solutions for regular data $\mu \in W^{-1,2}$; we refer to Section 4 for more details. Such solutions are unique for the problems considered here. For simplicity we shall refer to solutions to Dirichlet problems of the type

$$
\begin{cases}-\operatorname{div} a(x, D u)=\mu & \text { in } \Omega, \\ u=0 & \text { on } \partial \Omega,\end{cases}
$$

while more general situations can be treated as well; for instance, when $\mu$ is an integrable function with enough integrability the same statement works for general local distributional solutions to [1.9), without assuming anything on the boundary values; see again Section 4 The following is proved in Section 4.2

Theorem 1.5 (Lipschitz continuity). Under the assumptions $(1.2)$ and $(1.10)$, let $u \in$ $W^{1,1}(\Omega)$ be the unique SOLA to 1.17 . Assume that

$$
\mathbf{I}_{1}^{|\mu|}(\cdot, R) \in L^{\infty}(\Omega) \quad \text { for every } R>0 .
$$

Then $D u \in L_{\text {loc }}^{\infty}\left(\Omega, \mathbb{R}^{n}\right)$. Moreover, there exists a constant c, depending only on $n, L / v$, $L_{1}, \sigma$ and $\operatorname{diam}(\Omega)$, such that for any ball $B_{2 R} \subseteq \Omega$,

$$
\|D u\|_{L^{\infty}\left(B_{R / 2}\right)} \leq c f_{B_{R}}|D u| d x+c\left\|\mathbf{I}_{1}^{|\mu|}(\cdot, R)\right\|_{L^{\infty}\left(B_{R}\right)}+c s L_{1} R^{\sigma} .
$$


In particular, following [10] where the Poisson equation case $[1.3]$ is treated, an immediate consequence of 1.5$]$ is the following Lorentz space regularity criterion:

$$
\mu \in L(n, 1) \Rightarrow D u \in L_{\mathrm{loc}}^{\infty}\left(\Omega, \mathbb{R}^{n}\right) .
$$

We refer to (4.13) below for the definition of Lorentz spaces and to [12] for further consequences of (1.19). Moreover, we observe that 1.19 ) also implies estimates in noninterpolation spaces such as Morrey spaces, of the type obtained in [27, 28, 29]; see also the forthcoming papers [16, 17]. We finally remark that a main point in Theorem 1.5 is the low regularity of the coefficients $x \mapsto a(x, \cdot)$, which are assumed to be only Hölder continuous, rather than Lipschitz. We indeed recall that, usually, getting Lipschitz estimates involves iterations of gradient level sets which follow by differentiating the equation; here no differentiation is possible since we only assume $(1.10)$. Instead, thinking of $(1.10)$ as a fractional differentiability of $x \mapsto a(x, \cdot)$, we overcome this point by a fractional iteration.

The results in this paper are part of a research program announced in [15]; further developments will appear in [16, 17].

\section{Fractional Caccioppoli inequalities}

Here we shall prove Theorem 1.3 . We want to emphasize that the main point here is not the precise degree of fractional differentiability of $D u$, but rather the fact that some differentiability is possible for $\mathrm{Du}$. Indeed, for our purposes, any positive differentiability exponent for $\mathrm{Du}$ will suffice in order to perform the fractional De Giorgi type iteration leading to Theorem 1.4 .

\subsection{Basic notation and conventions}

In the following, we shall denote in the standard way

$$
B\left(x_{0}, R\right)=\left\{x \in \mathbb{R}^{n}:\left|x-x_{0}\right|<R\right\},
$$

the open ball with center $x_{0}$ and radius $R>0$; when not important or clear from the context, we shall simply write $B_{R} \equiv B\left(x_{0}, R\right)$, especially when the center is the origin. By $c$ we shall denote a generic constant, larger than one, and possibly varying in different occurrences; relevant dependence upon parameters will be indicated in parentheses or with special symbols such as $\tilde{c}$. Finally, for $A$ being a measurable subset with positive measure, and $g: A \rightarrow \mathbb{R}^{k}$ being a measurable map, we shall denote

$$
f_{A} g(x) d x:=\frac{1}{|A|} \int_{A} g(x) d x .
$$

In this and in the next section, we shall always argue under the assumptions of Theorem 1.2. we therefore remark that $\mu$ denotes here an $L^{\infty}$-function, and that we shall use the standard notation $|\mu|(A):=\|\mu\|_{L^{1}(A)}$ whenever $A \subset \Omega$ is a measurable subset. We shall use the converse notation: whenever $g \in L^{1}(\Omega)$, we shall denote $|g|(A):=$ $\|g\|_{L^{1}(A)}$. 


\subsection{Fractional Sobolev spaces and difference operators}

For a bounded open set $A \subset \mathbb{R}^{n}$ and $k \in \mathbb{N}$, parameters $\alpha \in(0,1)$ and $q \in$ $[1, \infty)$, the fractional Sobolev space $W^{\alpha, q}\left(A, \mathbb{R}^{k}\right)$ consists of those measurable mappings $w: A \rightarrow \mathbb{R}^{k}$ such that the following Gagliardo-type norm is finite:

$$
\begin{aligned}
\|w\|_{W^{\alpha, q}(A)} & :=\left(\int_{A}|w(x)|^{q} d x\right)^{1 / q}+\left(\int_{A} \int_{A} \frac{|w(x)-w(y)|^{q}}{|x-y|^{n+\alpha q}} d x d y\right)^{1 / q} \\
& =:\|w\|_{L^{q}(A)}+[w]_{\alpha, q ; A} .
\end{aligned}
$$

We also let

$$
[w]_{0, q ; A}:=\left(\int_{A}|w(x)|^{q} d x\right)^{1 / q} \text { and }[w]_{1, q ; A}:=\left(\int_{A}|D w(x)|^{q} d x\right)^{1 / q} .
$$

For a possibly vector valued function $w: \Omega \rightarrow \mathbb{R}^{k}$, and $h \in \mathbb{R}$, we define the finite difference operator $\tau_{i, h}$ for $i \in\{1, \ldots, n\}$ as

$$
\tau_{i, h} w(x) \equiv \tau_{i, h}(w)(x):=w\left(x+h e_{i}\right)-w(x),
$$

where $\left\{e_{i}\right\}_{1 \leq i \leq n}$ denotes the standard basis of $\mathbb{R}^{n}$. This makes sense whenever $x, x+$ $h e_{i} \in A$, an assumption that will be satisfied whenever we use $\tau_{i, h}$ in the following. In particular, we shall very often take $x \in A$ where $A \Subset \Omega$ is an open subset of $\Omega$, and $|h| \leq \operatorname{dist}(A, \partial \Omega)$. Not surprisingly, one can use finite difference operators to gain information about fractional Sobolev spaces:

Lemma 2.1. Let $w \in L^{q}\left(\Omega, \mathbb{R}^{k}\right), q \geq 1$, and assume that for $\bar{\alpha} \in(0,1], S \geq 0$ and an open set $\Omega^{\prime \prime} \Subset \Omega$ we have

$$
\left\|\tau_{i, h} w\right\|_{L^{q}\left(\Omega^{\prime \prime}\right)} \leq S|h|^{\bar{\alpha}}
$$

for every $i \in\{1, \ldots, n\}$ and every $h \in \mathbb{R}$ satisfying $0<|h| \leq d$, where $0<d \leq$ $\min \left\{1, \operatorname{dist}\left(\Omega^{\prime \prime}, \partial \Omega\right)\right\}$. Then $w \in W_{\text {loc }}^{\alpha, q}\left(\Omega^{\prime}, \mathbb{R}^{k}\right)$ for every $\alpha \in(0, \bar{\alpha})$ and each open set $\Omega^{\prime} \Subset \Omega^{\prime \prime}$, and there exists a constant $c \equiv c\left(d, \bar{\alpha}-\alpha, \operatorname{dist}\left(\Omega^{\prime}, \partial \Omega^{\prime \prime}\right), \operatorname{diam}(\Omega)\right)$, independent of $S$ and $w$, such that

$$
\|w\|_{W^{\alpha, q}\left(\Omega^{\prime}, \mathbb{R}^{k}\right)} \leq c\left(S+\|w\|_{L^{q}\left(\Omega^{\prime \prime}, \mathbb{R}^{k}\right)}\right) .
$$

\subsection{A priori full differentiability}

Let us start with a lemma on reverse Hölder inequalities that will be useful at several points.

Lemma 2.2. Let $g, h: A \rightarrow \mathbb{R}^{k}$ be integrable maps such that

$$
\left(f_{B_{R}}|g|^{\chi_{0}} d x\right)^{1 / \chi_{0}} \leq c f_{B_{2 R}}|g| d x+c_{1} R\left(f_{B_{R}}|h|^{q} d x\right)^{1 / q}+c_{1} R^{\sigma}
$$


whenever $B_{2 R} \subseteq A$, where $A \subset \mathbb{R}^{n}$ is an open subset, and $\chi_{0}, q>1, c, c_{1} \geq 0$. Then for every $t \in(0,1]$ there exists a constant $c_{0} \equiv c_{0}(n, c, t)$ such that, for every $B_{2 R} \Subset A$,

$\left(f_{B_{R}}|g|^{\chi_{0}} d x\right)^{1 / \chi_{0}} \leq c_{0}\left(f_{B_{2 R}}|g|^{t} d x\right)^{1 / t}+c_{0} c_{1} R\left(f_{B_{2 R}}|h|^{q} d x\right)^{1 / q}+c_{0} c_{1} R^{\sigma}$.

Proof. The proof is a modification of a well-known interpolation/covering argument usually applied for reverse Hölder inequalities, and it can be obtained with minor modifications from [18, Remark 6.12].

Definition 2.1. Denoting $z \equiv\left(z_{1}, \ldots, z_{n}\right) \in \mathbb{R}^{n}$, we define the globally Lipschitz functions

$$
W_{k}^{\xi}(z):=(|z \xi|-k)_{+}=\max \{|z \xi|-k, 0\}=T_{k}(|z \xi|)
$$

whenever $\xi \in\{1, \ldots, n\}$ and $k \geq 0$, and where, obviously, we have denoted the truncation operator as $T_{k}(t):=\max \{t-k, 0\}$, for $t, k \in \mathbb{R}$.

Here we consider an energy solution $v \in W^{1,2}(A)$ to the homogeneous equation

$$
\operatorname{div} a\left(x_{0}, D v\right)=0
$$

in a bounded domain $A \subset \Omega$, where $x_{0} \in A$. The vector field $z \mapsto a\left(x_{0}, z\right)$ is here taken to be the one appearing in (1.9) and therefore satisfying $(1.10)$.

Remark 2.1. We notice that by standard use of the ellipticity in $[1.2$, Young's inequality and (1.10), the following growth and coercivity conditions:

$$
|a(x, z)| \leq L(1+|z|), \quad\langle a(x, z), z\rangle+c s^{2} \geq c^{-1}|z|^{2}, \quad c \equiv c(n, L / v),
$$

are satisfied for every $x \in A$ and $z \in \mathbb{R}^{n}$, so that the natural domain of definition of the operator in 2.8 is $W^{1,2}(A)$.

Proposition 2.1. Let $v \in W^{1,2}(A)$ be a weak solution to 2.8 under the assumptions 1.2 , and let $k \geq 0$. Then $W_{k}^{\xi}(D v) \in W_{\mathrm{loc}}^{1,2}(A)$, and there exists $c \equiv c(n, L / v)$ such that

$$
\int_{B_{R / 2}}\left|D W_{k}^{\xi}(D v)\right| d x \leq \frac{c}{R} \int_{B_{R}}\left|W_{k}^{\xi}(D v)\right| d x
$$

whenever $B_{R} \subset A$.

Proof. We start by observing that, via a standard scaling and translation argument we may reduce the proof to the case $B_{R} \equiv B_{1}$; more precisely, we consider the function $y \mapsto R^{-1} v\left(x_{0}+R y\right)$ instead of $v(x)$ (see also the proof of Theorem 1.3). Accordingly, we shall first prove that

$$
\int_{B_{1 / 2}}\left|D W_{k}^{\xi}(D v)\right|^{2} d x \leq c \int_{B_{1}}\left|W_{k}^{\xi}(D v)\right|^{2} d x
$$


for $c \equiv c(n, L / v)$. To this end we recall that under the present assumptions standard regularity theory [18, Chapter 8$]$ implies

$$
D v \in W_{\mathrm{loc}}^{1,2}\left(B_{1}, \mathbb{R}^{n}\right) \cap L_{\mathrm{loc}}^{\infty}\left(B_{1}, \mathbb{R}^{n}\right) .
$$

Here, as well as in the following, we shall adopt the double index summation convention. In the weak form of (2.8),

$$
\int_{B_{1}} a_{i}\left(x_{0}, D v\right) D_{i} \varphi d x=0
$$

which holds whenever $\varphi$ is a smooth cut-off function with compact support in $B_{1}$, we take $D_{\xi} \varphi$ for $\xi \in\{1, \ldots, n\}$ instead of $\varphi$; after integration by parts we get

$$
\int_{B_{1}} \partial_{z_{j}} a_{i}\left(x_{0}, D v\right) D_{j} D_{\xi} v D_{i} \varphi d x=0 .
$$

In 2.13 we now take the test function

$$
\varphi:=\eta^{2} \frac{D_{\xi} v}{\left|D_{\xi} v\right|} W_{k}^{\xi}(D v),
$$

where $\eta$ is a smooth, non-negative cut-off function with compact support in $B_{1}$. Such a choice is admissible in (2.13), this being a consequence of (1.2) and (2.12); indeed 2.13 remains valid whenever we take $\varphi \in W_{0}^{1,2}\left(B_{1}\right)$ by 2.12, while again 2.12 and the Lipschitz continuity of the function $t \mapsto(t /|t|) T_{k}(|t|)$ for $k \geq 0$ imply that the test function $\varphi$ in 2.14 in fact belongs to $W_{0}^{1,2}\left(B_{1}\right)$. Therefore we get

$$
\begin{aligned}
\int_{B_{1}} \eta^{2} \partial_{z_{j}} a_{i}\left(x_{0}, D v\right) D_{j} D_{\xi} v D_{i} D_{\xi} v \frac{W_{k}^{\xi}(D v)}{\left|D_{\xi} v\right|} d x \\
+\int_{B_{1}} \eta^{2} \partial_{z_{j}} a_{i}\left(x_{0}, D v\right) \frac{D_{j} D_{\xi} v D_{\xi} v D_{i}\left(\left|D_{\xi} v\right|\right)}{\left|D_{\xi} v\right|} T_{k}^{\prime}\left(\left|D_{\xi} v\right|\right) d x \\
-\int_{B_{1}} \eta^{2} \partial_{z_{j}} a_{i}\left(x_{0}, D v\right) \frac{D_{j} D_{\xi} v D_{\xi} v D_{i}\left(\left|D_{\xi} v\right|\right)}{\left|D_{\xi} v\right|} \frac{W_{k}^{\xi}(D v)}{\left|D_{\xi} v\right|} d x \\
+2 \int_{B_{1}} \eta \partial_{z_{j}} a_{i}\left(x_{0}, D v\right) D_{j} D_{\xi} v D_{i} \eta D_{\xi} v \frac{W_{k}^{\xi}(D v)}{\left|D_{\xi} v\right|} d x=0 .
\end{aligned}
$$

By using the relations

$$
D_{j}\left(\left|D_{\xi} v\right|\right)=\frac{D_{j} D_{\xi} v D_{\xi} v}{\left|D_{\xi} v\right|} \quad \text { and } \quad \frac{D_{j} D_{\xi} v D_{\xi} v}{\left|D_{\xi} v\right|} D_{i}\left(\left|D_{\xi} v\right|\right)=D_{j} D_{\xi} v D_{i} D_{\xi} v
$$

we see that the sum of the first and the third term in 2.15 is zero, and therefore assumptions 1.2 allow us to estimate

$$
\int_{B_{1} \cap\left\{\left|D_{\xi} v\right|>k\right\}} \eta^{2}\left|D D_{\xi} v\right|^{2} d x \leq c \int_{B_{1} \cap\left\{\left|D_{\xi} v\right|>k\right\}} \eta|D \eta|\left|D D_{\xi} v\right|\left|W_{k}^{\xi}(D v)\right| d x
$$


so that by Young's inequality we conclude that

$$
\int_{B_{1} \cap\left\{\left|D_{\xi} v\right|>k\right\}} \eta^{2}\left|D D_{\xi} v\right|^{2} d x \leq c\|D \eta\|_{L^{\infty}}^{2} \int_{B_{1}}\left|W_{k}^{\xi}(D v)\right|^{2} d x .
$$

Choosing $\eta$ such that $\eta=1$ on $B_{1 / 2}$, and using $|D| D_{\xi} v|| \leq\left|D D_{\xi} v\right|$, yields

$$
\begin{aligned}
\int_{B_{1 / 2}}\left|D W_{k}^{\xi}(D v)\right|^{2} d x & \leq \int_{B_{1}}\left|D\left(\eta W_{k}^{\xi}(D v)\right)\right|^{2} d x \\
& \leq c \int_{B_{1}}|D \eta|^{2}\left|W_{k}^{\xi}(D v)\right|^{2} d x+c \int_{B_{1} \cap\left\{\left|D_{\xi} v\right|>k\right\}} \eta^{2}\left|D D_{\xi} v\right|^{2} d x
\end{aligned}
$$

so that 2.11) follows by taking 2.16) into account. Now we denote by $\chi>1$ the usual Sobolev embedding exponent, i.e. $\chi:=2 n /(n-2)$ if $n>2$, while $\chi>2$ if $n=2$. Using the classical Sobolev embedding theorem together with 2.11 ) we get

$$
\left\|W_{k}^{\xi}(D v)\right\|_{L^{x}\left(B_{1 / 2}\right)} \leq c\left\|W_{k}^{\xi}(D v)\right\|_{W^{1,2}\left(B_{1 / 2}\right)} \leq c\left\|W_{k}^{\xi}(D v)\right\|_{L^{2}\left(B_{1}\right)} .
$$

By rescaling back the previous inequality to a general ball $B_{R} \subset A$ (recall that our original domain is $A$ ) we obtain the usual reverse Hölder type inequality

$$
\left(f_{B_{R / 2}}\left|W_{k}^{\xi}(D v)\right|^{\chi} d x\right)^{1 / \chi} \leq c\left(f_{B_{R}}\left|W_{k}^{\xi}(D v)\right|^{2} d x\right)^{1 / 2}
$$

whenever $B_{R} \subset A$. Applying Lemma 2.2 with $g=\left|W_{k}^{\xi}(D v)\right|^{2}, \chi_{0}:=\chi / 2>1, t=1$, $c_{1}=0$, and then Hölder's inequality, we get

$$
\left(f_{B_{R / 2}}\left|W_{k}^{\xi}(D v)\right|^{2} d x\right)^{1 / 2} \leq c f_{B_{R}}\left|W_{k}^{\xi}(D v)\right| d x
$$

for any $B_{R} \subset A$; here we have used again that $\chi>2$. On the other hand, scaling back inequality 2.11) we obtain

$$
\int_{B_{R / 4}}\left|D W_{k}^{\xi}(D v)\right|^{2} d x \leq \frac{c}{R^{2}} \int_{B_{R / 2}}\left|W_{k}^{\xi}(D v)\right|^{2} d x .
$$

Finally, using Hölder's inequality together with the last two estimates yields

$$
\begin{aligned}
\int_{B_{R / 4}}\left|D W_{k}^{\xi}(D v)\right| d x & \leq c R^{n / 2}\left(\int_{B_{R / 4}}\left|D W_{k}^{\xi}(D v)\right|^{2} d x\right)^{1 / 2} \\
& \leq c R^{n / 2-1}\left(\int_{B_{R / 2}}\left|W_{k}^{\xi}(D v)\right|^{2} d x\right)^{1 / 2} \leq \frac{c}{R} \int_{B_{R}}\left|W_{k}^{\xi}(D v)\right| d x
\end{aligned}
$$

We have therefore obtained 2.10 with $R / 2$ replaced by $R / 4$. The final statement follows again by a standard covering argument. Again a covering argument and 2.17 imply that $W_{k}^{\xi}(D v) \in W_{\mathrm{loc}}^{1,2}(A)$, and the proof is complete. 


\subsection{Fractional differentiability}

The fractional differentiability of $W_{k}^{\xi}(D u)$ can be obtained by combining the differentiability estimate of Proposition 2.1 with a suitable comparison estimate between the original solution and the solution to a homogeneous problem with frozen coefficients. For $B_{2 R} \equiv B\left(x_{0}, 2 R\right) \subset \Omega$, denote by $w \in u+W_{0}^{1,2}\left(B_{2 R}\right)$ the unique solution to the homogeneous Dirichlet problem

$$
\begin{cases}\operatorname{div} a(x, D w)=0 & \text { in } B_{2 R} \\ w=u & \text { on } \partial B_{2 R}\end{cases}
$$

We similarly define $v \in w+W_{0}^{1,2}\left(B_{R}\right)$ to be the unique solution to the homogeneous Dirichlet problem with frozen coefficients

$$
\begin{cases}\operatorname{div} a\left(x_{0}, D v\right)=0 & \text { in } B_{R} \\ v=w & \text { on } \partial B_{R} .\end{cases}
$$

First we observe that [27, Lemma 4.1] applied with $\theta=n, p=2$ and $q=p-1$ gives

Lemma 2.3. Let $u$ be as in Theorem 1.2 and $w \in u+W_{0}^{1,2}\left(B_{2 R}\right)$ be defined as in 2.18. Then for a constant $c$ depending only on $n, v$,

$$
\int_{B_{2 R}}|D u-D w| d x \leq c R|\mu|\left(B_{2 R}\right) .
$$

Then we compare $u$ and $v$ as follows:

Lemma 2.4. Let $u$ be as in Theorem 1.2 and let $w \in u+W_{0}^{1,2}\left(B_{2 R}\right)$ and $v \in$ $w+W_{0}^{1,2}\left(B_{R}\right)$ be defined as in 2.18 and 2.19 respectively. Then for a constant $c$ depending only on $n, L / v$,

$$
\int_{B_{R}}|D u-D v| d x \leq R\left(1+L_{1} R^{\sigma}\right)|\mu|\left(B_{2 R}\right)+c L_{1} R^{\sigma} \int_{B_{2 R}}(s+|D u|) d x .
$$

Proof. By growth and coercivity conditions in 2.9) we may apply Gehring's lemma in the version presented in [18, Chapter 6] to find there exists a constant $\chi_{0}>2$ such that the reverse type inequality

$$
\left(f_{B_{Q / 2}}(s+|D w|)^{\chi_{0}} d x\right)^{1 / \chi_{0}} \leq c\left(f_{B_{\varrho}}(s+|D w|)^{2} d x\right)^{1 / 2}
$$

holds whenever $B_{Q} \subseteq B_{2 R}$ is a ball not necessarily concentric with $B_{2 R}$, for a constant $c$ depending only on $n, L / v$. Therefore, a further application of Lemma 2.2 with $g \equiv s+|D w|$, an application of Hölder's inequality, and finally a standard covering argument, shows that also

$$
\left(f_{B_{R}}(s+|D w|)^{2} d x\right)^{1 / 2} \leq c f_{B_{2 R}}(s+|D w|) d x
$$


Now we recall [27, Lemma 4.6]: a completely similar—and actually standard—comparison argument leads to the estimate

$$
\int_{B_{R}}|D w-D v|^{2} d x \leq c L_{1}^{2} R^{2 \sigma} \int_{B_{R}}(s+|D w|)^{2} d x,
$$

where $c$ depends on $n, L / v$. The previous inequality and 2.22 yield

$$
\begin{aligned}
\int_{B_{R}}|D w-D v| d x & \leq c R^{n / 2}\left(\int_{B_{R}}|D w-D v|^{2} d x\right)^{1 / 2} \\
& \leq c L_{1} R^{n / 2+\sigma}\left(\int_{B_{R}}(s+|D w|)^{2} d x\right)^{1 / 2} \\
& \leq c L_{1} R^{\sigma} \int_{B_{2 R}}(s+|D w|) d x,
\end{aligned}
$$

and 2.21 follows from the previous inequality and 2.20).

As a consequence of definition 2.7), and of the fact that the truncation operator $t \mapsto T_{k}(t)$ is globally Lipschitz with Lipschitz constant equal to one, the inequality

$$
\left|W_{k}^{\xi}\left(z_{2}\right)-W_{k}^{\xi}\left(z_{1}\right)\right| \leq\left|z_{2}-z_{1}\right|
$$

holds whenever $z_{2}, z_{1} \in \mathbb{R}^{n}$, so that 2.21 in turn implies

$$
\int_{B_{R}}\left|W_{k}^{\xi}(D u)-W_{k}^{\xi}(D v)\right| d x \leq c R|\mu|\left(B_{2 R}\right)+c L_{1} R^{\sigma} \int_{B_{2 R}}(s+|D u|) d x,
$$

where the constant $c$ depends only on $n, L / v, L_{1}$, whenever $R \leq 1$; this last assumption is only used to estimate $L_{1} R^{\sigma} \leq L_{1}$.

We now state a first form of the fractional Caccioppoli inequality.

Proposition 2.2. Under the assumptions of Theorem 1.2. whenever $\xi \in\{1, \ldots, n\}$, $k \geq 0$, and $\Omega^{\prime} \Subset \Omega$ is an open subset, the inequality

$$
\left[W_{k}^{\xi}(D u)\right]_{\sigma_{0}, 1 ; \Omega^{\prime}} \leq c \int_{\Omega} W_{k}^{\xi}(D u) d x+c|\mu|(\Omega)+c L_{1}(s+|D u|)(\Omega)
$$

holds for every $\sigma_{0}<\sigma /(1+\sigma)$, where the constant $c$ depends only on the quantities $n$, $L / v, L_{1}, \sigma_{0}, \operatorname{dist}\left(\Omega^{\prime}, \partial \Omega\right)$ and $\operatorname{diam}(\Omega)$.

Proof. We shall revisit some techniques of [22, 23, 27]. Keeping for the rest of the proof $\xi \in\{1, \ldots, n\}$ and $k \geq 0$ fixed, let us take a ball $B \Subset \Omega$; we denote by $Q_{\text {inn }} \equiv Q_{\text {inn }}(B)$ the largest cube, concentric with $B$ and with sides parallel to the coordinate axes, contained in $B$. The cube $Q_{\text {inn }}(B)$ will be called the inner cube of $B$. Now we select an intermediate open subset $\Omega^{\prime \prime} \Subset \Omega$, in the sense that $\Omega^{\prime} \Subset \Omega^{\prime \prime} \Subset \Omega$ and $\operatorname{dist}\left(\Omega^{\prime}, \partial \Omega^{\prime \prime}\right) \approx \operatorname{dist}\left(\Omega^{\prime \prime}, \partial \Omega\right) \approx \operatorname{dist}\left(\Omega^{\prime}, \partial \Omega\right)$; we set $d_{1}:=\min \left\{\operatorname{dist}\left(\Omega^{\prime}, \partial \Omega^{\prime \prime}\right), \operatorname{dist}\left(\Omega^{\prime \prime}, \partial \Omega\right), \operatorname{dist}\left(\Omega^{\prime}, \partial \Omega\right)\right\}$. We choose $h \in \mathbb{R}$ satisfying

$$
0<|h| \leq \min \left\{\left(d_{1} / 16\right)^{1+\sigma}, 1 / 16\right\}=: \tilde{d} .
$$


We take $x_{0} \in \Omega^{\prime \prime}$, and fix a ball of radius $|h|^{1 /(1+\sigma)}$ as follows:

$$
B \equiv B(h)=B\left(x_{0},|h|^{1 /(1+\sigma)}\right) .
$$

By 2.26 we have $8 B \subset \Omega$. Let us first define $w \in u+W_{0}^{1,2}(8 B)$, and then $v \in$ $w+W_{0}^{1,2}(4 B)$, as the unique solutions to the following Dirichlet problems:

$$
\begin{cases}\operatorname{div} a(x, D w)=0 & \text { in } 8 B \\ w=u & \text { on } \partial(8 B)\end{cases}
$$

and

$$
\begin{cases}\operatorname{div} a\left(x_{0}, D v\right)=0 & \text { in } 4 B \\ v=w & \text { on } \partial(4 B)\end{cases}
$$

respectively; we intend to apply the comparison estimates found for the problems (2.18)2.19]. Using that $|h| \leq \tilde{d}$ as prescribed in 2.26 , which implies $|h| \leq|h|^{1 /(1+\sigma)}$, it follows that

$$
h e+B \subset 2 B \quad \text { if } e \in \mathbb{R}^{n} \text { and }|e|=1,
$$

so that we may estimate

$$
\begin{aligned}
& \int_{B}\left|\tau_{i, h} W_{k}^{\xi}(D u)\right| d x \leq c \int_{B}\left|\tau_{i, h} W_{k}^{\xi}(D v)\right| d x \\
&+c \int_{B}\left|W_{k}^{\xi}\left(D u\left(x+h e_{i}\right)\right)-W_{k}^{\xi}\left(D v\left(x+h e_{i}\right)\right)\right| d x \\
&+c \int_{B}\left|W_{k}^{\xi}(D u)-W_{k}^{\xi}(D v)\right| d x \\
& \leq c \int_{B}\left|\tau_{i, h} W_{k}^{\xi}(D v)\right| d x+c \int_{2 B}\left|W_{k}^{\xi}(D u)-W_{k}^{\xi}(D v)\right| d x
\end{aligned}
$$

Again by 2.28 and using standard properties of $W^{1,1}$-maps together with estimate 2.10, we have

$$
\int_{B}\left|\tau_{i, h} W_{k}^{\xi}(D v)\right| d x \leq|h| \int_{2 B}\left|D W_{k}^{\xi}(D v)\right| d x \leq c|h|^{\sigma /(1+\sigma)} \int_{4 B}\left|W_{k}^{\xi}(D v)\right| d x .
$$

By 2.24] we have

$\int_{4 B}\left|W_{k}^{\xi}(D u)-W_{k}^{\xi}(D v)\right| d x \leq c|h|^{1 /(1+\sigma)}|\mu|(8 B)+c L_{1}|h|^{\sigma /(1+\sigma)} \int_{8 B}(s+|D u|) d x$,

and therefore, since $|h| \leq 1$ and $\sigma \leq 1$ imply $|h|^{1 / 1+\sigma} \leq|h|^{\sigma /(1+\sigma)}$, we also have

$$
\int_{4 B}\left|W_{k}^{\xi}(D u)-W_{k}^{\xi}(D v)\right| d x \leq c|h|^{\sigma /(1+\sigma)}\left[|\mu|(8 B)+L_{1} \int_{8 B}(s+|D u|) d x\right] .
$$


Using this last estimate together with 2.30 in 2.29) yields

$$
\begin{aligned}
& \int_{B}\left|\tau_{i, h} W_{k}^{\xi}(D u)\right| d x \\
& \quad \leq c|h|^{\sigma /(1+\sigma)}\left[\int_{8 B}\left|W_{k}^{\xi}(D u)\right| d x+|\mu|(8 B)+L_{1} \int_{8 B}(s+|D u|) d x\right] .
\end{aligned}
$$

Now we conclude with a covering argument: we first take a lattice of cubes $\left\{Q_{j}\right\}_{j \leq J}$ with sidelength equal to $2|h|^{1 /(1+\sigma)} / \sqrt{n}$, centered at points $\left\{x_{j}\right\} \subset \Omega^{\prime \prime}$, with sides parallel to the coordinate axes, and such that

$$
\left|\Omega^{\prime \prime} \backslash \bigcup Q_{j}\right|=0, \quad Q_{m} \cap Q_{j} \neq \emptyset \Leftrightarrow m=j .
$$

In turn, such cubes can be viewed as inner cubes of a family of balls in the sense that $Q_{j} \equiv Q_{\text {inn }}\left(B_{j}\right)$ and $B_{j} \equiv B\left(x_{j},|h|^{1 /(1+\sigma)}\right)$, as considered in 2.27), and we sum up inequalities 2.31) for $j \leq J$ and $B \equiv B_{j}$ to get

$$
\begin{aligned}
& \sum \int_{Q_{j}}\left|\tau_{i, h} W_{k}^{\xi}(D u)\right| d x \\
& \leq c|h|^{\sigma /(1+\sigma)} \sum\left[\int_{8 B_{j}}\left|W_{k}^{\xi}(D u)\right| d x+|\mu|\left(8 B_{j}\right)+L_{1} \int_{8 B_{j}}(s+|D u|) d x\right] .
\end{aligned}
$$

By construction, and in particular by 2.26 , we have $8 B_{j} \subset \Omega$ for every $j \leq J$. Moreover by 2.32 each of the dilated balls $8 B_{j}$ intersects the similar ones $8 B_{k}$ fewer than $(64 \sqrt{n})^{n}$ times; therefore the family $\left\{8 B_{j}\right\}$ has the finite intersection property. Using this observation, 2.32 and 2.33 give

$$
\begin{aligned}
& \int_{\Omega^{\prime \prime}}\left|\tau_{i, h} W_{k}^{\xi}(D u)\right| d x \\
& \quad \leq c|h|^{\sigma /(1+\sigma)}\left[\int_{\Omega}\left|W_{k}^{\xi}(D u)\right| d x+|\mu|(\Omega)+L_{1} \int_{\Omega}(s+|D u|) d x\right] .
\end{aligned}
$$

Since $i$ was arbitrary in $\{1, \ldots, n\}$, we are now in a position to apply Lemma 2.1, with $q=1$ : estimate 2.5 applied together with 2.34 gives 2.25) whenever $\sigma_{0}<\sigma /(1+\sigma)$, as desired. The proof is complete.

Proof of Theorem 1.3 The proof relies on the previous result, and we can obviously take $\sigma=1$ since no dependence on the $x$-variable appears in $a(\cdot)$ in this case. We directly prove the Caccioppoli inequality for the most general case $[1.9]$, that is,

$$
\begin{aligned}
{\left[\left(\left|D_{\xi} u\right|-k\right)_{+}\right]_{\sigma_{0}, 1 ; B_{R / 2}} \leq } & \frac{c}{R^{\sigma_{0}}} \int_{B_{R}}\left(\left|D_{\xi} u\right|-k\right)_{+} d x \\
& +c R^{1-\sigma_{0}}|\mu|\left(B_{R}\right)+c L_{1} R^{\sigma-\sigma_{0}}(s+|D u|)\left(B_{R}\right),
\end{aligned}
$$


so that Theorem 1.3 follows by taking $L_{1}=0$. Let $B_{R} \equiv B\left(x_{0}, R\right)$ be the ball in question; we use the following scaling:

$\tilde{u}(y):=R^{-1} u\left(x_{0}+R y\right), \quad \tilde{a}(y, z):=a\left(x_{0}+R y, z\right), \quad \tilde{\mu}(y):=R \mu\left(x_{0}+R y\right)$,

and we observe that $\tilde{u} \in W^{1,2}\left(B_{1}\right)$ solves the new equation

$$
-\operatorname{div} \tilde{a}(y, D \tilde{u})=\tilde{\mu} \quad \text { weakly in } B_{1} .
$$

Moreover we observe that the newly defined vector field $\tilde{a}(\cdot)$ satisfies assumptions $\sqrt{1.2}$ with $x$ frozen - and 1.10 with $L_{1}$ replaced by $L_{1} R^{\sigma}$. Therefore writing 2.25 for $\tilde{u}$ and $\tilde{\mu}$, and taking $\Omega^{\prime} \equiv B_{1 / 2}$, we get

$$
\left[W_{k}^{\xi}(D \tilde{u})\right]_{\sigma_{0}, 1 ; B_{1 / 2}} \leq c \int_{B_{1}} W_{k}^{\xi}(D \tilde{u}) d x+c|\tilde{\mu}|\left(B_{1}\right)+c L_{1} R^{\sigma}(s+|D \tilde{u}|)\left(B_{1}\right),
$$

and inequality (2.35) follows from the previous one by scaling back to $u$ and $\mu$, and using the fact that $\left[W_{k}^{\xi}(D \tilde{u})\right]_{\sigma_{0}, 1 ; B_{1 / 2}}=R^{\sigma_{0}-n}\left[W_{k}^{\xi}(D u)\right]_{\sigma_{0}, 1 ; B_{R / 2}}$.

Remark 2.2. Theorem 1.3 still holds for general solutions to 1.9 for problems with measure data; see Theorem 4.2 below.

Remark 2.3. Inequality 1.12 of course also holds at the $L^{2}$-level:

$$
\left[\left(\left|D_{\xi} u\right|-k\right)_{+}\right]_{\sigma_{0}, 2 ; B_{R / 2}} \leq \frac{c}{R^{2 \sigma_{0}}} \int_{B_{R}}\left(\left|D_{\xi} u\right|-k\right)_{+}^{2} d x+\frac{c R^{2}}{R^{2 \sigma_{0}}} \int_{B_{R}}|\mu|^{2} d x,
$$

and this remains valid for general distributional solutions $u \in W^{1,2}(\Omega)$ provided $\mu \in$ $L^{2}(\Omega)$; see Section 4.1 below. It is indeed sufficient to use 2.10 instead of 2.17, and then to replace inequality 2.20$)$ by

$$
\int_{B_{2 R}}|D u-D w|^{2} d x \leq c R^{2} \int_{B_{2 R}}|\mu|^{2} d x .
$$

This last inequality follows simply by testing (2.18) and (1.9) in $B_{2 R}$ with $u-w$ and using monotonicity in a standard way; finally, with (2.23) at our disposal we can repeat the same argument of Proposition 2.2 and eventually obtain (2.38) by the scaling argument of Theorem 1.3 .

\section{Proof of the pointwise a priori estimates}

In view of the a priori $W^{\sigma_{0}, 1}$-estimate of Proposition 2.2 let us recall that the fractional version of the Sobolev embedding theorem (see [5, Theorem 7.34]; here we need to take $\Omega^{\prime}$ smooth enough, a domain satisfying for instance the cone condition is suitable) states that

$$
\left\|W_{k}^{\xi}(D u)\right\|_{L^{t}\left(\Omega^{\prime}\right)} \leq c\left(\Omega^{\prime}\right)\left\|W_{k}^{\xi}(D u)\right\|_{W^{\sigma_{0}, 1}\left(\Omega^{\prime}\right)}
$$


where the exponent $t$ can be taken to satisfy

$$
1<t \leq \frac{n}{n-\sigma_{0}}, \quad \text { where } \quad 0<\sigma_{0}<\frac{\sigma}{1+\sigma}
$$

The combination of 3.1 and 2.25 gives

$$
\left\|W_{k}^{\xi}(D u)\right\|_{L^{t}\left(\Omega^{\prime}\right)} \leq c\left\|W_{k}^{\xi}(D u)\right\|_{L^{1}(\Omega)}+c|\mu|(\Omega)+c L_{1}(s+|D u|)(\Omega) .
$$

The same inequality of course applies after replacing $\Omega$ by a general subdomain, since all the estimates up to now were derived using only the fact that $u$ is a local weak solution. The constant $c$ appearing in 3.3 depends on $n, L / \nu$, $\operatorname{dist}\left(\Omega^{\prime}, \partial \Omega\right)$ and $\Omega^{\prime}$, but in the following, when applying inequality 3.3 , we shall always have $\Omega^{\prime} \equiv B_{1}$, so that the dependence of $c$ in the relevant estimates will be finally only on $n, L / v$.

Lemma 3.1. Let $u$ be as in Theorem 1.2 and let $k \geq 0, d>0$ and $2 B \subseteq \Omega$ be a ball with radius $2 R$; let $\xi \in\{1, \ldots, n\}$. Finally, assume that

$$
\left|B \cap\left\{\left|D_{\xi} u\right|>k\right\}\right| \leq \frac{1}{d} \int_{B} W_{k}^{\xi}(D u) d x .
$$

Then there exists a constant $c \equiv c\left(n, L / v, L_{1}\right)$ such that

$$
\begin{aligned}
\left(\frac{1}{d R^{n}} \int_{B} W_{k}^{\xi}(D u) d x\right)^{\varepsilon} \leq & \frac{c}{d R^{n}} \int_{2 B} W_{k}^{\xi}(D u) d x \\
& +\frac{c|\mu|(2 B)}{d R^{n-1}}+\frac{c L_{1}(s+|D u|)(2 B)}{d R^{n-\sigma}}
\end{aligned}
$$

where $\varepsilon \in(0,1)$ is a constant depending only on $n, \sigma_{0}$.

Proof. Let $B \equiv B\left(x_{0}, R\right)$ for some $x_{0} \in \Omega$. We start by observing that we can reduce to the case $B \equiv B_{1}$ by the rescaling procedure in 2.36-2.37; this time $y \in B_{2}$ and needless to say if (3.4) holds for $u$ on $B$, then it holds for $\tilde{u}$ on $B_{1}$. Next, by means of a similar scaling argument we reduce to the case $d=1$. Indeed, we now let $v(x):=d^{-1} u(x)$, and observe that $v$ weakly solves $-\operatorname{div} \tilde{a}(x, D v)=\tilde{\mu}$, where $\tilde{a}(x, z):=d^{-1} a(x, d z)$ and $\tilde{\mu}:=d^{-1} \mu$, while the new vector field $\tilde{a}(\cdot)$ still satisfies 1.2 and 1.10 with $s$ replaced by $s / d$. Writing estimate (3.5) for $v$-in the case when $d=1$ - and $k$ replaced by $k / d$, and with respect to the new operator $\tilde{a}(\cdot)$, we obtain

$$
\begin{aligned}
\left(\frac{1}{R^{n}} \int_{B} W_{k / d}^{\xi}(D v) d x\right)^{\varepsilon} \leq & \frac{c}{R^{n}} \int_{2 B} W_{k / d}^{\xi}(D v) d x \\
& +\frac{c|\tilde{\mu}|(2 B)}{R^{n-1}}+\frac{c L_{1}[(s / d)+|D v|](2 B)}{R^{n-\sigma}}
\end{aligned}
$$

which immediately yields (3.5) by taking into account the definition of $v$, and observing that $W_{k / d}^{\xi}(D v)=W_{k}^{\xi}(D u) / d$. We will therefore prove the lemma in the special case 
$B \equiv B_{1}$ and $d=1$; moreover we assume that $\left|B_{1} \cap\left\{\left|D_{\xi} u\right|>k\right\}\right|>0$, otherwise 3.5 trivializes. Similarly, we may also assume that

$$
\int_{B_{1}} W_{k}^{\xi}(D u) d x>0,
$$

and therefore, taking $t>1$ as in (3.2) and again using (3.4), we have

$$
\begin{aligned}
\left(\int_{B_{1}} W_{k}^{\xi}(D u) d x\right)^{1 / t} & =\left(\int_{B_{1}} W_{k}^{\xi}(D u) d x\right)^{1 / t-1} \int_{B_{1}} W_{k}^{\xi}(D u) d x \\
& \leq\left|B_{1} \cap\left\{\left|D_{\xi} u\right|>k\right\}\right|^{1 / t-1} \int_{B_{1}} W_{k}^{\xi}(D u) d x .
\end{aligned}
$$

In turn, applying Hölder's inequality yields

$$
\int_{B_{1}} W_{k}^{\xi}(D u) d x \leq\left|B_{1} \cap\left\{\left|D_{\xi} u\right|>k\right\}\right|^{1-1 / t}\left(\int_{B_{1}}\left(W_{k}^{\xi}(D u)\right)^{t} d x\right)^{1 / t} .
$$

The last two inequalities give now

$$
\left(\int_{B_{1}} W_{k}^{\xi}(D u) d x\right)^{1 / t} \leq\left(\int_{B_{1}}\left(W_{k}^{\xi}(D u)\right)^{t} d x\right)^{1 / t}
$$

On the other hand, by 3.3 written for $\Omega^{\prime} \equiv B_{1}$ and $\Omega \equiv B_{2}$, we gain

$$
\left(\int_{B_{1}}\left(W_{k}^{\xi}(D u)\right)^{t} d x\right)^{1 / t} \leq c \int_{B_{2}} W_{k}^{\xi}(D u) d x+c|\mu|\left(B_{2}\right)+c(s+|D u|)\left(B_{2}\right),
$$

so that combining (3.6) and 3.7 gives 3.5 with $B \equiv B_{1}$ and $d=1$, taking $\varepsilon:=$ $1 / t<1$.

Remark 3.1 (Reverse Hölder inequality). For simplicity we consider the case with no dependence on $x$ in the vector field $a(\cdot)$; therefore scaling back to $B_{2 R}$ in inequality (3.7), we gain the following reverse Hölder inequality on level sets, which is well known in the homogeneous case $\mu=0$ :

$$
\left(f_{B_{R}}\left(W_{k}^{\xi}(D u)\right)^{t} d x\right)^{1 / t} \leq c f_{B_{2 R}} W_{k}^{\xi}(D u) d x+c R f_{B_{2 R}}|\mu| d x
$$

Proof of Theorems 1.1 and 1.2 We give the proof of Theorem 1.2 since Theorem 1.1 follows by taking $L_{1}=0$, which we can when no dependence on $x$ is allowed. We shall follow the De Giorgi type iteration argument brilliantly introduced in [21]. Let us select $\xi \in\{1, \ldots, n\}$; we consider a dyadic sequence of radii $R_{j}:=2^{1-j} R, j \geq 0$, and let $B_{j}:=B\left(x_{0}, R_{j}\right)$. We define $k_{0}:=0$ and, recursively for $j \geq 0$,

$$
k_{j+1}:=k_{j}+\frac{1}{\delta R_{j}^{n}} \int_{B_{j+1}} W_{k_{j}}^{\xi}(D u) d x .
$$


The number $\delta \in(0,1)$ will be chosen shortly; it is going to be suitably small, but will always depend just on $n, L / v$. We note that $\left\{k_{j}\right\}$ is a non-decreasing sequence. Our next aim is to prove the estimate

$$
k_{j+1}-k_{j} \leq \frac{1}{2}\left(k_{j}-k_{j-1}\right)+\frac{c|\mu|\left(B_{j}\right)}{R_{j}^{n-1}}+\frac{c L_{1}(s+|D u|)\left(B_{j}\right)}{R_{j}^{n-\sigma}}
$$

for every $j \in \mathbb{N}$, and for a constant $c$ only depending on $n, L / \nu$. In order to prove 3.9 for $j \in \mathbb{N}$ we first observe that we may assume $k_{j+1}>k_{j}$, as otherwise 3.9 is trivially satisfied; then we start by showing that

$$
\delta^{\varepsilon} \leq c \delta \frac{k_{j}-k_{j-1}}{k_{j+1}-k_{j}}+\frac{c|\mu|\left(B_{j}\right)}{d_{j} R_{j}^{n-1}}+\frac{c L_{1}(s+|D u|)\left(B_{j}\right)}{d_{j} R_{j}^{n-\sigma}}
$$

for the corresponding $j \in \mathbb{N}$, with a constant $c$ depending only on $n, L / \nu$, and for a positive sequence $\left\{d_{j}\right\}$ to be defined in a few lines. Here $\varepsilon \in(0,1)$ is the number introduced in Lemma 3.1. We have

$$
\begin{aligned}
\left|B_{j} \cap\left\{\left|D_{\xi} u\right|>k_{j}\right\}\right| & \leq \frac{1}{k_{j}-k_{j-1}} \int_{B_{j} \cap\left\{\left|D_{\xi} u\right|>k_{j}\right\}} W_{k_{j-1}}^{\xi}(D u) d x \\
& \leq \frac{1}{k_{j}-k_{j-1}} \int_{B_{j}} W_{k_{j-1}}^{\xi}(D u) d x \\
& =\delta R_{j-1}^{n}=2^{n} \delta R_{j}^{n}=\frac{2^{n}}{k_{j+1}-k_{j}} \int_{B_{j+1}} W_{k_{j}}^{\xi}(D u) d x .
\end{aligned}
$$

Observe that we repeatedly used (3.8). From the last chain of inequalities it is clear that choosing $\delta$ small enough in order to have $\delta \leq 2^{-(n+1)} R_{j}^{-n}\left|B_{j}\right|$-which imposes on $\delta$ a smallness condition depending only on $n$-we obtain

$$
\left|B_{j} \cap\left\{\left|D_{\xi} u\right|>k_{j}\right\}\right| \leq \frac{1}{2}\left|B_{j}\right| \text {. }
$$

At this point we define

$$
d_{j}:=\frac{k_{j+1}-k_{j}}{2^{n}}
$$

so that we are able to apply Lemma 3.1 with the choice $k \equiv k_{j}, d \equiv d_{j}$ and $B \equiv B_{j+1}$ as assumption (3.4) turns out to be satisfied. This yields

$$
\begin{aligned}
& \left(\frac{1}{d_{j} R_{j}^{n}} \int_{B_{j+1}} W_{k_{j}}^{\xi}(D u) d x\right)^{\varepsilon} \\
& \quad \leq \frac{c}{d_{j} R_{j}^{n}} \int_{B_{j}} W_{k_{j}}^{\xi}(D u) d x+\frac{c|\mu|\left(B_{j}\right)}{d_{j} R_{j}^{n-1}}+\frac{c L_{1}(s+|D u|)\left(B_{j}\right)}{d_{j} R_{j}^{n-\sigma}} .
\end{aligned}
$$

In turn, using (3.8) again we observe that

$$
\frac{c}{d_{j} R_{j}^{n}} \int_{B_{j}} W_{k_{j}}^{\xi}(D u) d x \leq \frac{c}{d_{j} R_{j}^{n}} \int_{B_{j}} W_{k_{j-1}}^{\xi}(D u) d x=c \delta \frac{d_{j-1}}{d_{j}} .
$$


Merging the last inequality with (3.13), and using (3.8) together with the definition of $d_{j}$ in 3.12 , we find

$$
\begin{aligned}
\delta^{\varepsilon} & \leq c\left(\frac{1}{d_{j} R_{j}^{n}} \int_{B_{j+1}} W_{k_{j}}^{\xi}(D u) d x\right)^{\varepsilon} \\
& \leq \frac{c}{d_{j} R_{j}^{n}} \int_{B_{j}} W_{k_{j}}^{\xi}(D u) d x+\frac{c|\mu|\left(B_{j}\right)}{d_{j} R_{j}^{n-1}}+\frac{c L_{1}(s+|D u|)\left(B_{j}\right)}{d_{j} R_{j}^{n-\sigma}} \\
& \leq c \delta \frac{k_{j}-k_{j-1}}{k_{j+1}-k_{j}}+\frac{c|\mu|\left(B_{j}\right)}{d_{j} R_{j}^{n-1}}+\frac{c L_{1}(s+|D u|)\left(B_{j}\right)}{d_{j} R_{j}^{n-\sigma}}
\end{aligned}
$$

for a constant $c$ which depends only on $n, L / v$ and $L_{1}$; the proof of 3.10$)$ is therefore complete. We can now show that $3.9 p$ holds. Indeed, if $k_{j+1}-k_{j} \leq(1 / 2)\left(k_{j}-k_{j-1}\right)$ holds then also (3.9) does, trivially. Otherwise, we have $\left(k_{j}-k_{j-1}\right) /\left(k_{j+1}-k_{j}\right)<2$, which, used in 3.14, yields

$$
\delta^{\varepsilon} \leq \tilde{c} \delta+\frac{c|\mu|\left(B_{j}\right)}{d_{j} R_{j}^{n-1}}+\frac{c L_{1}(s+|D u|)\left(B_{j}\right)}{d_{j} R_{j}^{n-\sigma}},
$$

with $\tilde{c}$ depending only on $n, L / \nu, L_{1}$. Therefore reducing further the size of $\delta \equiv$ $\delta\left(n, L / v, L_{1}\right)$ in order to have $\delta<(1 / 2 \tilde{c})^{1 /(1-\varepsilon)}$, and recalling the choice of $d_{j}$, we conclude that

$$
k_{j+1}-k_{j} \leq \frac{c|\mu|\left(B_{j}\right)}{R_{j}^{n-1}}+\frac{c L_{1}(s+|D u|)\left(B_{j}\right)}{R_{j}^{n-\sigma}}
$$

so that 3.9 follows in any case. The proof of (1.8) now also follows by iterating (3.9):

$$
\begin{aligned}
& k_{m}-k_{1} \leq k_{m+1}-k_{1}=\sum_{j=1}^{m}\left(k_{j+1}-k_{j}\right) \\
& \leq \frac{1}{2} \sum_{j=1}^{m}\left(k_{j}-k_{j-1}\right)+c \sum_{j=1}^{m} \frac{|\mu|\left(B_{j}\right)}{R_{j}^{n-1}}+c L_{1} \sum_{j=1}^{m} \frac{(s+|D u|)\left(B_{j}\right)}{R_{j}^{n-\sigma}} \\
& \leq \frac{1}{2} k_{m}+c \sum_{j=1}^{\infty} \frac{|\mu|\left(B_{j}\right)}{R_{j}^{n-1}}+c L_{1} \sum_{j=1}^{\infty} \frac{(s+|D u|)\left(B_{j}\right)}{R_{j}^{n-\sigma}} \\
& \leq \frac{1}{2} k_{m}+c \mathbf{I}_{1}^{|\mu|}\left(x_{0}, 2 R\right)+c L_{1}\left[\mathbf{I}_{\sigma}^{|D u|}\left(x_{0}, 2 R\right)+\frac{s}{\sigma} R^{\sigma}\right] \text {. }
\end{aligned}
$$

Therefore we have

$$
\lim _{m \rightarrow \infty} k_{m} \leq 2 k_{1}+c \mathbf{I}_{1}^{|\mu|}\left(x_{0}, 2 R\right)+c L_{1}\left[\mathbf{I}_{\sigma}^{|D u|}\left(x_{0}, 2 R\right)+\frac{s}{\sigma} R^{\sigma}\right] .
$$


On the other hand, since $D u$ is here assumed to be continuous, and since 3.11 implies that $\inf _{B_{m}}|D u| \leq k_{m}$, we have

$$
\begin{aligned}
\left|D_{\xi} u\left(x_{0}\right)\right| & =\lim _{m \rightarrow \infty} \inf _{B_{m}}\left|D_{\xi} u\right| \\
& \leq \lim _{m \rightarrow \infty} k_{m} \\
& \leq 2 k_{1}+c \mathbf{I}_{1}^{|\mu|}\left(x_{0}, 2 R\right)+c L_{1}\left[\mathbf{I}_{\sigma}^{|D u|}\left(x_{0}, 2 R\right)+\frac{s}{\sigma} R^{\sigma}\right],
\end{aligned}
$$

where $c$ depends only $n, L / v, L_{1}$, and it remains to estimate $k_{1}$. By (3.8), for a constant $c$ depending on $n, L / v, L_{1}$ via the use of the number $\delta$ which in turn depends only on $n, L / v, L_{1}$, we have

$$
k_{1} \leq c f_{B\left(x_{0}, R\right)}\left|D_{\xi} u\right| d x .
$$

Using this last inequality in 3.15 , and recalling that $\xi \in\{1, \ldots, n\}$ is arbitrary, finally gives (1.8) and the proof is complete.

Proof of Theorem 1.4 We first observe that Lemma 3.1 follows with $D_{\xi} u$ replaced by $w$ and $L_{1}=0$. Indeed, note that (3.3) and the subsequent Lemma 3.1 do not necessitate the general form of 2.25), while an inequality formulated on balls as (1.12) is sufficient. We just observe that the scaling argument in the proof of Lemma $3.1-$ necessary to reduce to the case when $B_{R} \equiv B_{1}$ - should be slightly modified as follows: we define $\tilde{u}$ as in (2.36) with $y \in B_{2}$, while, since we are now dealing with a measure, we define the scaled Radon measure $\tilde{\mu}(A):=R^{1-n} \mu\left(x_{0}+R A\right)$ whenever $A \subset \mathbb{R}^{n}$ is a Borel set-recall that we consider $\mu$ as defined on the whole $\mathbb{R}^{n}$. In this way it is easy to check that the couple $(\tilde{u}, \tilde{\mu})$ satisfies 1.12 on $B_{R} \equiv B_{2}$. We therefore argue as in Lemma 3.1 to deduce (3.5) with $w \equiv D_{\xi} u$ and $L_{1}=0$. In turn this is everything we need to repeat the argument of the proof of Theorems $1.1+1.2$ with $w \equiv D_{\xi} u$, and estimate 1.16 finally follows. Note that the assertion concerning the validity of 1.16 only at the Lebesgue points of $w$ is due to the fact that here $w$ is not necessarily continuous, and therefore the convergence in the first line of 3.15 - where again $w \equiv D_{\xi} u$ - holds only if $x_{0}$ is a Lebesgue point of $w$.

Remark 3.2. The result of Theorems $1.1+1.2$ can also be considered as the higher order analog of that in [23, 31] when $p=2$. There the following bound is proved:

$$
u\left(x_{0}\right) \leq c\left(f_{B\left(x_{0}, R\right)} u^{\gamma} d x\right)^{1 / \gamma}+c \mathbf{I}_{2}^{\mu}\left(x_{0}, 2 R\right), \quad \gamma>1,
$$

for non-negative solutions in the case $\mu$ is a non-negative measure, and also in the case the vector field $a(\cdot)$ has a non-differentiable structure. This is of course the proper non-linear analog of the classical estimate $|u(x)| \leq c I_{2}(|\mu|)(x)$ valid for solutions to 1.3 . The result of Theorem 1.1 indeed upgrades estimate 3.16 to the gradient level, and extends it to the case of arbitrary signed measures. 


\section{Selected consequences}

Here we show that estimates $(1.8)$ and $(1.11)$ extend to solutions to general measure data problems. In the second part of this section we prove Theorem 1.5 , while in the last part we give an example on how to derive sharp and local a priori estimates in some function spaces starting from estimates (1.8) and (1.11). We shall not give of course all the possible applications; we shall rather present a sample of them, while along the lines presented here other applications can be deduced.

\subsection{General measure data}

By 1.7 , the smoothing properties of $\mathbf{I}_{\beta}^{\mu}(\cdot, R)$ are exactly those of the classical Riesz potential. We denote by $\mathcal{M}(\Omega)$ the space of signed Radon measures with finite total mass on $\Omega$, while $M^{m}(\Omega)$ denotes the usual Marcinkiewicz space-also called weak- $L^{m}$ space-i.e. the space of those measurable (vector) functions $f$ such that

$$
\|f\|_{M^{m}(\Omega)}^{m}:=\sup \lambda^{m}|\{x \in \Omega:|f(x)|>\lambda\}|<\infty, \quad m>0 .
$$

From (1.7]—see [4, Lemma 3.1.1]—whenever $B_{\varrho} \subseteq \Omega$, it follows that

$$
\left\|\mathbf{I}_{\beta}^{\mu}(\cdot, \varrho / 2)\right\|_{L^{n m /(n-m \beta)}\left(B_{\varrho / 2}\right)} \leq c\|\mu\|_{L^{m}\left(B_{\varrho}\right)}
$$

for every $\mu \in L_{\text {loc }}^{m}(\Omega)$ where $m>1$, while

$$
\left\|\mathbf{I}_{\beta}^{\mu}(\cdot, \varrho / 2)\right\|_{M^{n /(n-\beta)}\left(B_{\varrho / 2}\right)} \leq c|\mu|\left(B_{\varrho}\right)
$$

for every $\mu \in \mathcal{M}(\Omega)$. Inequalities 4.1$]-4.2$ can be easily obtained from [4, Theorem 3.1.4] via a standard localization argument-passing from $\mu$ to $\mu\left\llcorner B_{\varrho}\right.$, and then using the classical boundedness properties of the Riesz potentials in $\mathbb{R}^{n}$.

We are now ready to establish estimates (1.8) and (1.11) without assuming $\mu \in$ $L^{\infty}(\Omega)$, and consequently, that $u \in C_{\mathrm{loc}}^{1, \alpha}(\Omega)$. Yet, as for measure data, for the sake of simplicity we shall confine ourselves to the case of homogeneous Dirichlet problems of the type (1.17), but more general cases can be considered as well. The existence of a solution to [1.17) is obtained in [8, 9] via an approximation method we briefly recall; this approximation method determines the class of Solutions Obtained by Limit of Approximations (SOLA). We consider a standard, symmetric and non-negative mollifier $\phi \in C_{0}^{\infty}\left(B_{1}\right)$ such that $\|\phi\|_{L^{1}\left(\mathbb{R}^{n}\right)}=1$, and then define, for $k \in \mathbb{N}, \phi_{k}(x):=k^{n} \phi(k x)$. Finally the functions $\mu_{k}: \mathbb{R}^{n} \rightarrow \mathbb{R}$ are defined via convolution, $\mu_{k}(x):=\left(\mu * \phi_{k}\right)(x)$ —see (4.6) below for the definition. Next, by standard monotonicity methods, we find a unique solution $u_{k} \in W_{0}^{1,2}(\Omega)$ to

$$
\begin{cases}-\operatorname{div} a\left(x, D u_{k}\right)=\mu_{k} & \text { in } \Omega, \\ u_{k}=0 & \text { on } \partial \Omega .\end{cases}
$$

Up to passing to a subsequence (not relabeled) we may assume that $\mu_{k} \rightarrow \mu$ weakly in the sense of measures, while the results in [8, 9] imply

$$
D u_{k} \rightarrow D u \quad \text { strongly in } L^{t}(\Omega) \text { for every } t<\frac{n}{n-1} \text {, and a.e., }
$$


so that (1.17) is solved by $u$ in the usual distributional sense, and therefore $u$ is a SOLA to (1.17). Moreover, by [6, Theorem 8.3] (see also [32, Theorem 4.1]), $u$ is the only SOLA of 1.17, in the sense that if $v \in W_{0}^{1,1}(\Omega)$ is a distributional solution to 1.17$\}_{1}$ obtainable as a pointwise limit of solutions $v_{k} \in W_{0}^{1,2}(\Omega)$ to problems of the type $\left[4.3\right.$ with $\mu_{k}$ replaced by $\tilde{\mu}_{k}$ and $\tilde{\mu}_{k} \rightarrow \mu$, then $u \equiv v$. Now, let us consider $B\left(x_{0}, R\right) \subset \Omega$, and let us keep $R$ fixed: we consider $x_{0} \rightarrow \mathbf{I}_{1}^{\left|\mu_{k}\right|}\left(x_{0}, 2 R\right) \equiv \mathbf{I}_{1}^{\left|\mu_{k}\right|}\left(x_{0}\right)$ as a pointwise function of $x_{0}$; it follows that

$$
\mathbf{I}_{1}^{\left|\mu_{k}\right|}\left(x_{0}\right) \leq\left(\mathbf{I}_{1}^{|\mu|} * \phi_{k}\right)\left(x_{0}\right) .
$$

Let us reproduce the proof of 4.5 for the reader's convenience; we recall that given two Radon measures $\mu, \lambda \in \mathcal{M}\left(\mathbb{R}^{n}\right)$, their convolution is defined by

$$
(\mu * \lambda)(A):=\iint \chi_{A}(x+y) d \mu(x) d \lambda(y)
$$

for any Borel set $A \subset \mathbb{R}^{n}$, where $\chi_{A}$ stands for the indicator function of $A$. Letting $\lambda:=\phi_{k}(x) d x$, we have

$$
\begin{aligned}
\left|\mu_{k}\right|\left(B\left(x_{0}, s\right)\right) & \leq \int_{B_{1}} \phi_{k}(y) \int_{\Omega+B_{1 / k}} \chi_{B\left(x_{0}, s\right)}(x+y) d|\mu|(x) d y \\
& \leq \int_{B_{1}} \phi_{k}(y)|\mu|\left(B\left(x_{0}-y, s\right)\right) d y .
\end{aligned}
$$

Therefore, using Fubini's theorem, 4.5 follows by estimating

$$
\begin{aligned}
\mathbf{I}_{1}^{\left|\mu_{k}\right|}\left(x_{0}\right) & \leq \int_{B_{1}} \phi_{k}(y) \int_{0}^{2 R} \frac{|\mu|\left(B\left(x_{0}-y, t\right)\right)}{t^{n-1}} \frac{d t}{t} d y \\
& =\int_{B_{1}} \phi_{k}(y) \mathbf{I}_{1}^{|\mu|}\left(x_{0}-y\right) d y=\left(\mathbf{I}_{1}^{|\mu|} * \phi_{k}\right)\left(x_{0}\right) .
\end{aligned}
$$

Again for a fixed $R$, since $\mathbf{I}_{1}^{|\mu|}(\cdot, 2 R) \in M^{n /(n-1)}(\Omega)$ by 4.2 , we recall that, up to extracting a subsequence depending on $R$, we have

$$
\left(\mathbf{I}_{1}^{|\mu|} * \phi_{k}\right)\left(x_{0}, 2 R\right) \rightarrow \mathbf{I}_{1}^{|\mu|}\left(x_{0}, 2 R\right)
$$

almost everywhere in $\Omega$; recall that we have extended $\mu$ to the whole $\mathbb{R}^{n}$. In the same way the strong convergence in (4.4), together with 4.1), implies that, again up to a nonrelabeled subsequence depending on $R$, we have

$$
\mathbf{I}_{\sigma}^{\left|D u_{k}\right|}\left(x_{0}, 2 R\right) \rightarrow \mathbf{I}_{\sigma}^{|D u|}\left(x_{0}, 2 R\right)
$$

for almost every point $x_{0}$ such that $B\left(x_{0}, 2 R\right) \subset \Omega$, and again up to a subsequence depending on $R$. We are now ready for the proof of (1.11); by standard regularity theory it follows that $u_{k} \in C_{\text {loc }}^{1, \alpha}(\Omega)$ for some $\alpha \equiv \alpha(n, L / \nu, \sigma)>0$, and therefore we may apply estimate (1.11) to $u_{k}$, which together with 4.5 gives

$$
\left|D_{\xi} u_{k}\left(x_{0}\right)\right| \leq c f_{B\left(x_{0}, R\right)}\left|D_{\xi} u_{k}\right| d x+c \mathbf{I}_{1}^{|\mu|}\left(x_{0}, 2 R\right)+c L_{1}\left[\mathbf{I}_{\sigma}^{\left|D u_{k}\right|}\left(x_{0}, 2 R\right)+\frac{s}{\sigma} R^{\sigma}\right] \text {. }
$$


Finally, estimate (1.11) for $u$ follows by letting $k \rightarrow \infty$ in the previous inequality, and taking 4.7)- 4.8 into account; note that since for every $R$ the extracted subsequences for which (4.7) and 4.8) hold depend on $R$, the convergence argument must be complemented by a standard diagonal argument made for radii $\left\{R_{m}\right\}_{m \in \mathbb{Z}}:=\left\{2^{-m} R\right\}_{m \in \mathbb{Z}}$, and using the fact that the functions $R \rightarrow \mathbf{I}_{\sigma}^{|\mu|}(\cdot, R)$ and $R \rightarrow \mathbf{I}_{\sigma}^{|D u|}(\cdot, R)$ are non-decreasing. Summarizing, we have proved the following:

Theorem 4.1. Under the assumptions 1.2 and 1.10 , let $u \in W_{\mathrm{loc}}^{1,1}(\Omega)$ be the unique SOLA to 1.17). Then there exists a constant $c \equiv c\left(n, L / v, L_{1}\right)$ such that the pointwise estimate

$$
\left|D u\left(x_{0}\right)\right| \leq c f_{B\left(x_{0}, R\right)}|D u| d x+c \mathbf{I}_{1}^{|\mu|}\left(x_{0}, 2 R\right)+c L_{1}\left[\mathbf{I}_{\sigma}^{|D u|}\left(x_{0}, 2 R\right)+\frac{s}{\sigma} R^{\sigma}\right]
$$

holds for almost every $x_{0} \in \Omega$ whenever $B\left(x_{0}, 2 R\right) \subseteq \Omega$.

Accordingly, keeping 2.35) in mind, with a much simpler proof since no potentials are now involved, we have

Theorem 4.2. Under the assumptions 1.2 and 1.10 , let $u \in W_{\mathrm{loc}}^{1,1}(\Omega)$ be the unique SOLA to (1.17). Then for every $B_{R} \subseteq \Omega$ and for every $\xi \in\{1, \ldots, n\}$,

$$
\begin{aligned}
{\left[\left(\left|D_{\xi} u\right|-k\right)_{+}\right]_{\sigma_{0}, 1 ; B_{R / 2}} \leq } & \frac{c}{R^{\sigma_{0}}} \int_{B_{R}}\left(\left|D_{\xi} u\right|-k\right)_{+} d x \\
& +c R^{1-\sigma_{0}}|\mu|\left(\overline{B_{R}}\right)+c L_{1} R^{\sigma-\sigma_{0}}(s+|D u|)\left(B_{R}\right)
\end{aligned}
$$

whenever $\sigma_{0}<\sigma /(1+\sigma)$, where the constant $c$ depends only on $n, L / v, L_{1}$ and $\sigma_{0}$. For solutions to (1.1) inequality (4.10) simplifies to (1.12).

Remark 4.1. We remark that in the case where $\mu \in L^{m}$ and $m$ is large enough to have $\mu \in W^{-1,2}$, i.e. $m \geq 2 n /(n+2)$, general local weak solutions can be considered by standard local approximation arguments, and we do not need to consider Dirichlet boundary value problems. In this case, given the original solution $u \in W^{1,2}(\Omega)$, the approximation considered goes via problems of the type

$$
\begin{cases}-\operatorname{div} a\left(x, D u_{k}\right)=\mu_{k} & \text { in } B \\ u_{k}=u & \text { on } \partial B\end{cases}
$$

where $B \subseteq \Omega$ is any fixed ball.

\subsection{Proof of Theorem 1.5}

First of all, we notice that by considering the approximation scheme used in Section 4.1 (see 4.3) or a similar one in the case of standard weak solutions outlined in Remark 4.1, we may reduce ourselves to the situation when $D u$ is locally bounded in $\Omega$, and then prove 1.19 as an a priori estimate. The details for this are at this point standard and the reader will have no difficulty checking them. Let us consider a ball $B_{2 R}$ and concentric 
balls $B_{R} \subset B_{\varrho} \subset B_{r} \subset B_{2 R}$. We apply estimate 1.11 with $R=(r-\varrho) / 2$, at the generic point $x_{0} \in B_{\varrho}$, thereby getting

$$
\begin{aligned}
\sup _{B_{\varrho}}|D u| \leq & \frac{c L_{1} R^{\sigma}}{\sigma} \sup _{B_{r}}|D u|+\frac{c}{(r-\varrho)^{n}} \int_{B_{2 R}}|D u| d x \\
& +c\left\|\mathbf{I}_{1}^{|\mu|}(\cdot, R)\right\|_{L^{\infty}\left(B_{2 R}\right)}+\frac{c s L_{1}}{\sigma} R^{\sigma},
\end{aligned}
$$

where, for every $y \in B_{\varrho}$, we have just estimated as follows:

$$
\mathbf{I}_{\sigma}^{|D u|}(y,(r-\varrho)) \leq\left(\int_{0}^{r} \frac{d t}{t^{1-\sigma}}\right) \sup _{B_{r}}|D u| \leq \frac{R^{\sigma}}{\sigma} \sup _{B_{r}}|D u| .
$$

We now select a positive radius $R_{0} \equiv R_{0}\left(n, L / v, L_{1}, \sigma\right)$ such that $c L_{1} R_{0}^{\sigma} / \sigma \leq 1 / 2$, and applying Lemma 4.1 below with the choice $\phi(r):=\sup _{B_{r}}|D u|$ we deduce estimate (1.18), under the additional condition that $R \leq R_{0}$.

In the case $R_{0} \leq R$, for every $\delta>0$ we select a ball $B\left(x_{0}, R_{0} / 4\right)$, with center in $B_{R}$, such that $\|D u\|_{L^{\infty}\left(B_{R}\right)} \leq\|D u\|_{L^{\infty}\left(B\left(x_{0}, R_{0} / 4\right)\right)}+\delta$; by construction we have $B\left(x_{0}, R_{0} / 2\right) \subset B_{2 R}$. Using estimate 1.18 on $B\left(x_{0}, R_{0} / 4\right)$ yields

$$
\begin{aligned}
\|D u\|_{L^{\infty}\left(B_{R}\right)} & \leq\|D u\|_{L^{\infty}\left(B\left(x_{0}, R_{0} / 4\right)\right)}+\delta \\
& \leq c\left(\frac{R}{R_{0}}\right)^{n} f_{B_{2 R}}|D u| d x+c\left\|\mathbf{I}_{1}^{|\mu|}(\cdot, R)\right\|_{L^{\infty}\left(B_{2 R}\right)}+\frac{c s L_{1}}{\sigma} R^{\sigma}+\delta .
\end{aligned}
$$

Taking into account that $R_{0}$ depends only on $n, L / \nu, L_{1}, \sigma$, inequality 1.18 for $B_{R}$ follows by letting $\delta \rightarrow 0$ in the last estimate.

Lemma 4.1 ([18, Chapter 6]). Let $\varphi:[R, 2 R] \rightarrow[0, \infty)$ be a bounded function such that

$$
\varphi(\varrho) \leq \frac{1}{2} \varphi(r)+\frac{\mathcal{B}}{(r-\varrho)^{n}}+K
$$

whenever $R<\varrho<r<2 R$, for fixed constants $\mathcal{B}, K \geq 0$. Then

$$
\varphi(R) \leq \frac{c \mathcal{B}}{R^{n}}+c K
$$

\subsection{Local integrability estimates}

Formula (1.8) allows us to prove in an easy way the optimal local form of the gradient integrability results obtained by Boccardo \& Gallouët [6, 7, 8, 9] for problems involving quasilinear equations with linear growth as well as those with the right hand side more integrable, as considered by Iwaniec [20]; see also [1, 14]. Local estimates in finer scales such as Lorentz or Orlicz spaces, and in general interpolation or rearrangement invariant function spaces, as for instance those of the type of Talenti [30], also follow as a corollary. Here we summarize some of the main integrability estimates achievable via Theorems $1.1,1.2$. For the sake of brevity we shall not state all the a priori estimates in 
a precise form; we shall give one example only which is going to emphasize that these can be achieved by combining estimate $(1.8)$ with an explicit a priori estimate available for the Riesz potentials, like for instance those recalled in 4.1)- 4.2). We anyway stress that the approach proposed here allows one to obtain explicit local a priori estimates in optimal form, something which is not easily achievable by the global methods presented in the literature for measure data problems [8, 9, 30], while alternative routes may involve lengthy procedures [27] not covering all cases. For further developments of our approach we refer to the forthcoming paper of Cianchi [12] where estimates like (1.8) play a central role.

As usual, we shall assume that $\mu$ is initially a Radon signed measure with finite total mass, i.e. $\mu \in \mathcal{M}(\Omega)$. For the SOLA $u$ of $(1.9)$ found in the previous section-and for general distributional solutions in the case where the right hand side is a function with enough integrability, that is, $\mu \in L^{m} \subset W^{-1,2}$ — the following holds in $\Omega$ :

$$
\left\{\begin{array}{l}
\mu \in \mathcal{M}(\Omega) \Rightarrow D u \in M_{\mathrm{loc}}^{n /(n-1)}, \\
\mu \in L \log L_{\mathrm{loc}} \Rightarrow D u \in L_{\mathrm{loc}}^{n /(n-1)}, \\
\mu \in L_{\mathrm{loc}}^{m} \text { for } 1<m<n \Rightarrow D u \in L_{\mathrm{loc}}^{n m /(n-m)}, \\
\mu \in M_{\mathrm{loc}}^{m} \text { for } 1<m<n \Rightarrow D u \in M_{\mathrm{loc}}^{n m /(n-m)}, \\
\mu \in L_{\mathrm{loc}}(m, q) \text { for } 1<m<n, 0<q<\infty \\
\Rightarrow D u \in L_{\mathrm{loc}}(n m /(n-m), q) .
\end{array}\right.
$$

The above implications are a straightforward consequence of estimate (1.8) and of the basic regularization properties of the Riesz potentials, as, for instance, those in (4.1) (4.2). We recall that the spaces appearing in the last line of (4.12) are Lorentz spaces: for $1 \leq m<\infty$ and $0<q \leq \infty$, a measurable map $g$ belongs to $L(m, q)(\Omega)$ iff

$$
\|g\|_{L(m, q)(\Omega)}^{q}:=q \int_{0}^{\infty}\left(\lambda^{m}|\{x \in \Omega:|g(x)|>\lambda\}|\right)^{q / m} \frac{d \lambda}{\lambda}<\infty
$$

when $q<\infty$; for $q=\infty$ we set $L(m, \infty)(\Omega) \equiv M^{m}(\Omega)$. The local variants of such spaces are then defined in the usual manner. Lorentz spaces are obtainable as interpolation spaces, and therefore the result in 4.125 follows via interpolation theorems by the linearity of the Riesz potential. Similar estimates can be obtained in Orlicz spaces via interpolation [11].

Example of a local estimate. We give the local version of the third inclusion from (4.12); similar local estimates can be derived for the others using (1.11). For SOLA or distributional solutions to 1.9 ,

$$
\left(f_{B_{R}}|D u|^{\frac{n m}{n-m}} d x\right)^{\frac{n-m}{n m}} \leq c f_{B_{2 R}}|D u| d x+c R\left(f_{B_{2 R}}|\mu|^{m} d x\right)^{1 / m}+c s L_{1} R^{\sigma}
$$

whenever $B_{2 R} \subseteq \Omega$, for a constant depending only on $n, m, L / \nu, L_{1}, \sigma$, where $\mu \in$ $L_{\text {loc }}^{m}(\Omega)$, with $m<n$. In proving 4.14 we can of course reduce to the case where $D u$ is locally bounded, such an additional assumption being removable by approximation. Let 
us consider $B_{2 R} \subseteq \Omega$; we then reduce to the case $B_{R} \equiv B_{1}$ via the scaling in 2.362.37). Next, we integrate (1.11) over $B_{1 / 2}$ with $R=1 / 2$, getting

$$
\begin{aligned}
\int_{B_{1 / 2}}|D u|^{\frac{n m}{n-m}} \leq & c\left(\int_{B_{1}}|D u| d x\right)^{\frac{n m}{n-m}}+c \int_{B_{1 / 2}} \mathbf{I}_{1}^{|\mu|}(x, 1 / 2)^{\frac{n m}{n-m}} d x \\
& +c \int_{B_{1 / 2}} \mathbf{I}_{\sigma}^{|D u|}(x, 1 / 2)^{\frac{n m}{n-m}} d x+c\left(s L_{1} / \sigma\right)^{\frac{n m}{n-m}} .
\end{aligned}
$$

Using (4.1) we get

$$
\left\|\mathbf{I}_{1}^{|\mu|}(\cdot, 1 / 2)\right\|_{L^{\frac{n m}{n-m}}\left(B_{1 / 2}\right)} \leq c\|\mu\|_{L^{m}\left(B_{1}\right)} .
$$

We now set

$$
\gamma:=\frac{n-m \sigma}{n-m}
$$

and notice that by the definition in 1.6 it follows that

$$
\mathbf{I}_{\sigma}^{|D u|}(x, 1 / 2)^{\frac{n m}{n-m}} \leq c(\sigma) \mathbf{I}_{\sigma}^{|D u|^{\gamma}}(x, 1 / 2)^{\frac{n m}{n-m \sigma}}
$$

where the constant $c(\sigma)>0$ blows up as $\sigma \rightarrow 0$. Indeed, we first notice that

$$
\int_{0}^{1 / 2} \frac{d t}{t^{1-\sigma}}=\frac{1}{\sigma}\left(\frac{1}{2}\right)^{\sigma}
$$

and observe that $\sigma \leq 1$ implies $\gamma \leq 1$; then, applying Jensen's inequality twice, we estimate

$$
\begin{aligned}
\mathbf{I}_{\sigma}^{|D u|}(x, 1 / 2)^{\frac{n m}{n-m}} & \leq c(n)\left(\frac{1}{\sigma}\right)^{\frac{n m}{n-m}}\left[f_{0}^{1 / 2} f_{B(x, t)}|D u(y)| d y \frac{d t}{t^{1-\sigma}}\right]^{\frac{\gamma n m}{n-m \sigma}} \\
& \leq c\left(\frac{1}{\sigma}\right)^{\frac{n m}{n-m} \frac{m(1-\sigma)}{n-m \sigma}}\left[f_{0}^{1 / 2} f_{B(x, t)}|D u(y)|^{\gamma} d y \frac{d t}{t^{1-\sigma}}\right]^{\frac{n m}{n-m \sigma}} \\
& \leq c(\sigma) \mathbf{I}_{\sigma}^{|D u|^{\gamma}}(x, 1 / 2)^{\frac{n m}{n-m \sigma}},
\end{aligned}
$$

so that $(4.18)$ follows. In turn, in view of (4.18), keeping (4.1) in mind, and using the definition (4.17), we have

$$
\left\|\mathbf{I}_{\sigma}^{|D u|}(\cdot, 1 / 2)\right\|_{L^{\frac{n m}{n-m}}\left(B_{1 / 2}\right)} \leq c(\sigma)\|D u\|_{L^{m \gamma}\left(B_{1}\right)} .
$$

Merging this last inequality and (4.18), and using also 4.16) with 4.15), we get

$$
\|D u\|_{L^{\frac{n m}{n-m}\left(B_{1 / 2}\right)}} \leq c\|D u\|_{L^{m \gamma}\left(B_{1}\right)}+c\|\mu\|_{L^{m}\left(B_{1}\right)}+c s L_{1} / \sigma .
$$

Scaling back the previous inequality as done for 2.36, we get

$$
\begin{aligned}
\left(f_{B_{R}}|D u|^{\frac{n m}{n-m}} d x\right)^{\frac{n-m}{n m}} \leq & c\left(f_{B_{2 R}}|D u|^{m \gamma} d x\right)^{\frac{1}{m \gamma}} \\
& +c R\left(f_{B_{2 R}}|\mu|^{m} d x\right)^{1 / m}+\frac{s L_{1}}{\sigma} R^{\sigma},
\end{aligned}
$$


which holds for any ball $B_{2 R} \subseteq \Omega$. Since $\sigma>0$, we also have that $m \gamma<n m /(n-m)$, and therefore we are able to apply Lemma 2.2 in its full strength, which in turn yields (4.14.

Acknowledgments. This research is supported by the ERC grant 207573 "Vectorial Problems", and by MIUR via the national project "Calcolo delle Variazioni". The author also thanks Verena Bögelein, Frank Duzaar and Jan Kristensen for several comments on a preliminary version of the manuscript and Andrea Cianchi for a useful conversation on regularity in borderline cases. Last but not least, the careful work of the referee is hereby acknowledged.

\section{References}

[1] Acerbi, E., Mingione, G.: Gradient estimates for a class of parabolic systems. Duke Math. J. 136, 285-320 (2007) Zbl 1113.35105 MR 2286632

[2] Adams, D. R.: A note on Riesz potentials. Duke Math. J. 42, 765-778 (1975) Zbl 0336.46038 MR 0458158

[3] Adams, D. R.: Lectures on $L^{p}$-potential theory. Department of Mathematics, Univ. of Umeå (1981)

[4] Adams, D. R., Hedberg, L. I.: Function Spaces and Potential Theory. Grundlehren Math. Wiss. 314, Springer, Berlin (1996) Zbl 0834.46021 MR 1411441

[5] Adams, R. A., Fournier, J. J. F.: Sobolev Spaces. 2nd ed., Pure Appl. Math. 140, Elsevier/Academic Press, Amsterdam (2003) Zbl 1098.46001 MR 2424078

[6] Boccardo, L.: Problemi differenziali ellittici e parabolici con dati misure. Boll. Un. Mat. Ital. A (7) 11, 439-461 (1997) Zbl 0893.35131 MR 1477785

[7] Boccardo, L.: Marcinkiewicz estimates for solutions of some elliptic problems with measure data. Ann. Mat. Pura Appl. (4) 188, 591-601 (2009) Zbl 1174.35038 MR 2533956

[8] Boccardo, L., Gallouët, T.: Nonlinear elliptic and parabolic equations involving measure data. J. Funct. Anal. 87, 149-169 (1989) Zbl 0707.35060 MR 1025884

[9] Boccardo, L., Gallouët, T.: Nonlinear elliptic equations with right-hand side measures. Comm. Partial Differential Equations 17, 641-655 (1992) Zbl 0812.35043 MR 1163440

[10] Cianchi, A.: Maximizing the $L^{\infty}$ norm of the gradient of solutions to the Poisson equation. J. Geom. Anal. 2, 499-515 (1992) Zbl 0780.35009 MR 1189042

[11] Cianchi, A.: An optimal interpolation theorem of Marcinkiewicz type in Orlicz spaces. J. Funct. Anal. 153, 357-381 (1998) Zbl 0913.46028 MR 1614590

[12] Cianchi, A.: Non-linear potentials, local solutions to elliptic equations and rearrangements. In preparation

[13] De Giorgi, E.: Sulla differenziabilità e l'analiticità delle estremali degli integrali multipli regolari. Mem. Accad. Sci. Torino Cl. Sci. Fis. Mat. Nat. (III) 125, no. 3, 25-43 (1957) Zbl 0084.31901 MR 0093649

[14] DiBenedetto, E., Manfredi, J. J.: On the higher integrability of the gradient of weak solutions of certain degenerate elliptic systems. Amer. J. Math. 115, 1107-1134 (1993) Zbl 0805.35037 MR 1246185

[15] Duzaar, F., Mingione, G.: Gradient estimates in non-linear potential theory. Rend. Lincei Mat. Appl. 20, 179-190 (2009) Zbl 1173.35065 MR 2506379

[16] Duzaar, F., Mingione, G.: Gradient estimates via non-linear potentials. Amer. J. Math., to appear

[17] Duzaar, F., Mingione, G.: Gradient continuity estimates. Calc. Var. Partial Differential Equations 39, 379-418 (2010) 
[18] Giusti, E.: Direct Methods in the Calculus of Variations. World Sci., River Edge, NJ (2003) Zbl 1028.49001 MR 1962933

[19] Hardy, G. H., Littlewood, J. E.: Some properties of fractional integrals. Math. Z. 27, 565-606 (1928) JFM 54.0275.05 MR 1544927

[20] Iwaniec, T.: Projections onto gradient fields and $L^{p}$-estimates for degenerated elliptic operators. Studia Math. 75, 293-312 (1983) Zbl 0552.35034 MR 0722254

[21] Kilpeläinen, T., Malý, J.: The Wiener test and potential estimates for quasilinear elliptic equations. Acta Math. 172, 137-161 (1994) Zbl 0820.35063 MR 1264000

[22] Kristensen, J., Mingione, G.: The singular set of $\omega$-minima. Arch. Ration. Mech. Anal. 177, 93-114 (2005) Zbl 1082.49036 MR 2187315

[23] Kristensen, J., Mingione, G.: The singular set of minima of integral functionals. Arch. Ration. Mech. Anal. 180, 331-398 (2006) Zbl 1116.49010 MR 2214961

[24] Ladyzhenskaya, O. A., Ural'tseva, N. N.: Linear and Quasilinear Elliptic Equations. Academic Press, New York (1968) Zbl 0164.13002 MR 0244627

[25] Malý, J., Ziemer, W. P.: Fine regularity of solutions of elliptic partial differential equations. Math. Surveys Monogr. 51, Amer. Math. Soc., Providence, RI (1997) Zbl 0882.35001 MR 1461542

[26] Mingione, G.: The singular set of solutions to non-differentiable elliptic systems. Arch. Ration. Mech. Anal. 166, 287-301 (2003) Zbl 1142.35391 MR 1961442

[27] Mingione, G.: The Calderón-Zygmund theory for elliptic problems with measure data. Ann Scuola Norm. Sup. Pisa Cl. Sci. (5) 6, 195-261 (2007) Zbl 1178.35168 MR 2352517

[28] Mingione, G.: Gradient estimates below the duality exponent. Math. Ann. 346, 571-627 (2010) Zbl 1193.35077 MR 2578563

[29] Mingione, G.: Nonlinear aspects of Calderón-Zygmund theory. Jahresber. Deutsch. Math.Verein. 112, 159-191 (2010) Zbl pre05824553

[30] Talenti, G.: Elliptic equations and rearrangements. Ann Scuola Norm. Sup. Pisa Cl. Sci. (4) 3, 697-717 (1976) Zbl 0341.35031 MR 0601601

[31] Trudinger, N. S., Wang, X. J.: On the weak continuity of elliptic operators and applications to potential theory. Amer. J. Math. 124, 369-410 (2002) Zbl 1067.35023 MR 1890997

[32] Trudinger, N. S., Wang, X. J.: Quasilinear elliptic equations with signed measure data. Discrete Contin. Dynam. Systems 23, 477-494 (2009) Zbl 1154.35351 MR 2449089 